\title{
Sensorless Whole-Body Compliance Control of Collaborative Manipulator Based on Haptic Filter and Position Controller
}

\author{
Binluan Wang $\mathbb{D}^{1},{ }^{1}$ Hongzhe Jin $\left(\mathbb{D},{ }^{1}\right.$ Jie Zhao $\mathbb{D}^{1},{ }^{1}$ Yubin Liu $\mathbb{D},{ }^{1}$ and Xiufang Liu ${ }^{2}$ \\ ${ }^{1}$ School of Mechatronics Engineering, Harbin Institute of Technology, Harbin 150080, China \\ ${ }^{2}$ Shanghai Aerospace Equipment Manufacturer Co., Ltd., Shanghai 200000, China \\ Correspondence should be addressed to Hongzhe Jin; hongzhejin@hit.edu.cn
}

Received 23 October 2020; Revised 3 April 2021; Accepted 9 May 2021; Published 24 May 2021

Academic Editor: Paul Honeine

Copyright (C) 2021 Binluan Wang et al. This is an open access article distributed under the Creative Commons Attribution License, which permits unrestricted use, distribution, and reproduction in any medium, provided the original work is properly cited.

This study presents a cost-effective sensorless whole-body compliance control strategy for collaborative manipulator. The control strategy realizes decoupled adjustable compliant effects, namely, stiffness, damping, and inertia controls, under a single control framework. The inherent position controller is retained, which ensures a smooth transition between normal position operation and compliance control. The two features can greatly simplify the customization of collaborative manipulator control algorithms. A modified sensorless disturbance observer based on generalized momentum is used to estimate the external torque, and this way eliminates the dependence on the force/torque sensors. Only basic motor position sensors are required. The compliant trajectory generated by the external torque is sufficiently smooth owing to the haptic filter. Various experiments prove that the modified sensorless disturbance observer is effective. The necessity of using the position servo loop for sensorless compliance control is discussed through a comparative experiment. The proposed compliance control strategy is further verified using sensorless and sensor-based disturbance observers.

\section{Introduction}

The application of robot manipulators outside the traditional industrial field has been increasing rapidly in the past few decades. The manipulators are required to interact with the changing environment and human in many conditions. Meanwhile, the compliance effects have gradually shifted from local compliance (only end-effector sensitive), such as polishing [1] and teaching [2, 3], to whole-body compliance (sensitive at any location of the manipulator), such as physical collision reaction [4] and collision reaction [5]. Many newly developed collaborative manipulator products are equipped with whole-body physical interaction capability, such as KUKA iiwa, Universal Robots, and YUMI. Some research adds passive elastic mechanical structure such as series elastic actuator into robot manipulator $[6,7]$ to realize compliant interaction, but this way complicates the design of the manipulator. Some researchers achieve good physical interaction performance by using various torque/ force sensors, such as joint torque sensors [8], tactile skin [9], force-sensitive end effector [10], and inertial measurement unit [11]. However, the cost of hardware increases at the same time. Therefore, the research on sensorless whole-body compliance control is important in developing a costeffective collaborative manipulator.

External force feedback is essential for compliance control. The motor current also couples with external force information when no sensors are used. An external force estimator based on disturbance observer is proposed in [12]. An estimation method that compares actual force calculated by the dynamics model with demanded force is proposed in [13]. The two methods eliminate the dependence on sensors but utilize joint acceleration signal that results in great noise. A real-time sensorless external torque estimator based on momentum observer was proposed by de Luca in [14] and proven in [15]. Fast and accurate joint space external torque estimation is realized only by torque command and joint velocity, without using acceleration signals. Kalman filter is combined with the momentum observer to estimate Cartesian external force in $[16,17]$. The momentum observer and its variants have been verified in considerable literature [18-21]. 
The study on compliance control has received considerable attention. Active compliance control is an effective way to realize interaction function while keeping the mechanical modification of manipulator at the lowest level. Impedance control proposed by Hogan is a classical algorithm to achieve compliance behavior [22]. Compared with other traditional control algorithms, such as hybrid force/ position control $[23,24]$, and parallel force/position control [25], impedance control has better robustness and does not require force measurement [26]. The controller receives position and outputs force. Applying force controller brings inconvenience in that the force controller is not always supported on manipulator products [27]. Admittance control considered as the inverse of impedance control converts external force into motion. Impedance control is realized while the low-level position controller is retained. In [28], momentum observer is combined with admittance control on a dual-arm robot to achieve sensorless compliance behavior. An adaptive neural network controller is used as low-level controller. In [29], a sensorless adaptive admittance controller is designed for industrial robots. The inherent low-level position controller is sustained, which helps the manipulator keep a consistent control structure with normal trajectory tasks. Other control methods such as sliding mode control techniques [30] and neural-networkbased adaptive control techniques [31] have been proposed by mainly focusing on the model uncertainties. A new integral sliding mode control strategy that can completely reject matched perturbation while not amplifying the unmatched one is proposed in [32]. This approach has a great potential in model uncertainty rejection.

Admittance control aims to control the impedance between the robot and the environment. Impedance consists of stiffness, damping, and inertia in general. Most studies on compliance control have applied second-order impedance that includes all three effects [28, 33-35]. The dynamics of the robot system can be manipulated at combined target stiffness, damping, and inertia effects. Other research on first-order impedance regarded as damping control (accommodation control) with stiffness and damping effects can be found in $[36,37]$. Zero-order impedance regarded as stiffness control with only stiffness effect is studied in [38], and it mostly aims at various stiffness controls. Pure damping control and inertia control (only damping or inertia is considered) have rarely been studied [27]. However, the two control methods have a great potential in physical interaction. As the inertia effect relates force and acceleration, pure inertia control makes the manipulator a floating mass body, which has a great potential in physical interaction, such as contact and collision simulation under zerogravity environment or aerospace noncooperative target capturing [39]. Pure damping control can quantificationally regulate velocity according to the contact force, which is helpful in precise velocity control during hands-on manipulation. Notably, a multifunctional compliance controller under admittance control structure can be realized using different components of the impedance.

On the basis of the idea discussed above, a cost-effective compliance control method for collaborative manipulators is proposed in this study. The strategy realizes three wholebody compliance control effects, namely, stiffness, damping, and inertia controls, under admittance control structure. The joints are approximated to quantificationally adjustable torsion spring, rotary damper, or rotary inertia. Only motor position sensors are required for acquisition of the joint space external torque owing to the momentum observer. No extra force/torque sensors are needed. A haptic feature filter is introduced to provide a smoother interaction experience and judge the convergence speed of the compliance controller. The control algorithm is studied in simulation environment and on a $2-\mathrm{DOF}$ manipulator.

The rest of the paper is organized as follows. Section 2 discusses the whole control strategy, including design of sensorless momentum observer and the design and analysis of the stiffness, damping, and inertia control algorithm. Section 3 proposes a simulation analysis of the compliance control strategy. Section 4 describes the verification results of sensorless and sensor-based momentum observer, as well as the proposed compliance control strategy. The defect of using torque controller for sensorless compliance control is also discussed. Conclusions and brief discussion of implementation complexity are presented in Section 5 .

\section{Compliance Control Strategy}

2.1. Framework of Control Strategy. As shown in Figure 1, the general control strategy is to map estimated external torque onto trajectory command without changing the inherent position controller. However, unlike traditional admittance control, the external contact force is not directly used to generate reference trajectory. Instead, we introduce a haptic filter to smoothen the estimated external torque first. Then, we map the filtered torque to the desired joint position, velocity, and acceleration features. After that, the reference trajectory commands are generated. In this way, the aforementioned spring, damper, and inertia haptic features can be achieved, respectively, through three control modes named stiffness, damping, and inertia controls. The manipulators can be abstracted as a series of torsion springs, dampers, and masses. Therefore, an appropriate design of the filter structure is required to represent the control effects and ease their tuning according to actual demands.

The joint space external torque is estimated by momentum observer, which ensures the global sensitivity of the robot manipulator. The active joint torque, which is an input of the momentum observer, can be obtained by using either torque sensors or motor current commands. Therefore, the compliance control strategy can be realized in either way.

2.2. Estimation of Joint Space External Torque. Sensorless estimation of joint space external torque is proposed based on the momentum observer in the previous work of De Luca et al. $[14,15]$. The momentum observer is modified using complete arm-plus-actuator dynamics in this study.

2.2.1. Dynamics Modeling with Actuator Dynamics. We consider the serial manipulator with $n$ rigid joints and define 


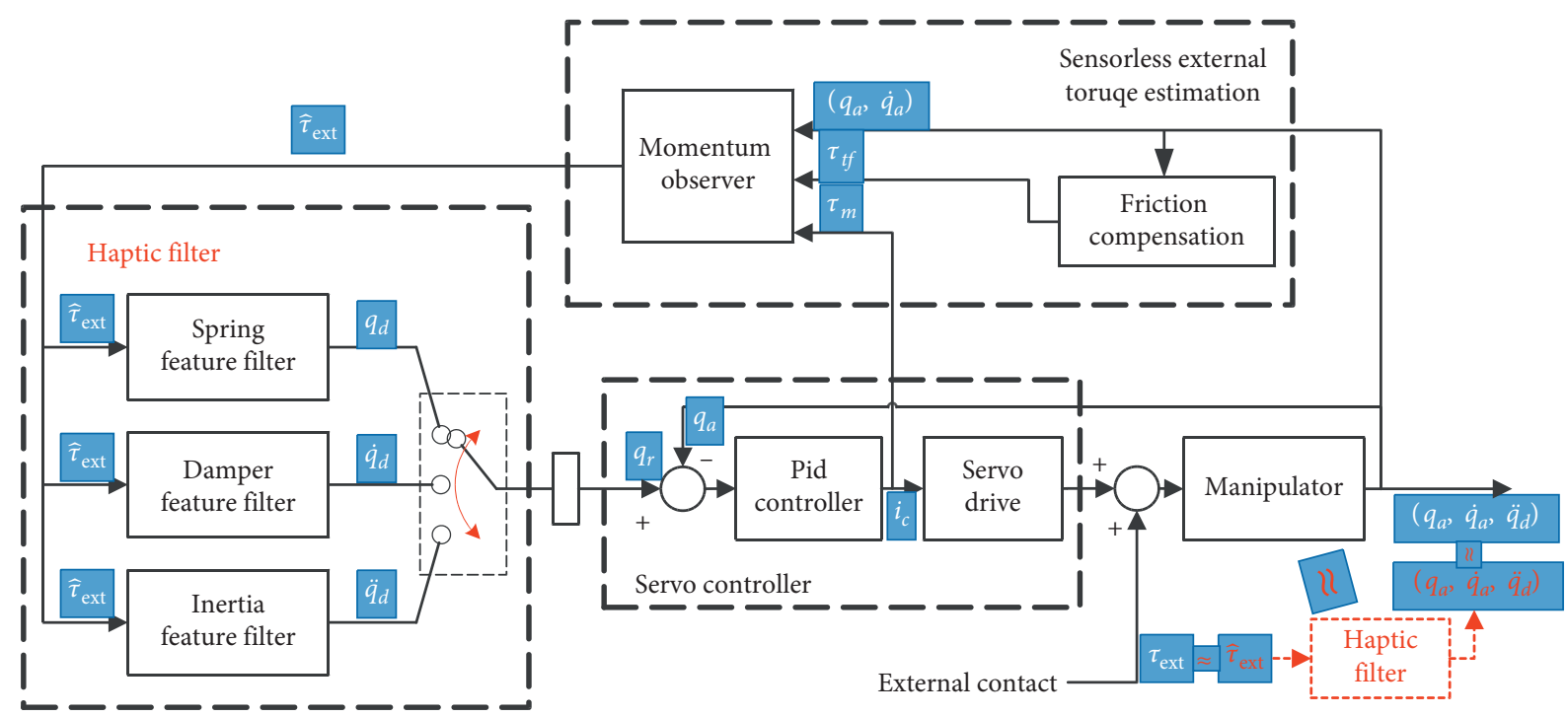

FIGURE 1: Sensorless compliance control strategy based on position controller. $\mathbf{q}_{\mathbf{d}}, \dot{q}_{\mathbf{d}}, \ddot{\mathbf{q}}_{\mathbf{d}}$ are the desired joint position, velocity, and acceleration, $\mathbf{q}_{r}$ is the reference trajectory, $\mathbf{i}_{c}$ is the current command, $\mathbf{q}_{a}, \dot{q}_{a}$ are the actual joint position and velocity, $\tau_{\mathrm{tf}}$ is the total friction compensation term, $\tau_{m}$ is the motor-side torque obtained from current command, and $\tau_{\text {ext }}, \widehat{\tau}_{\text {ext }}$ are the actual value and the estimation of external torque.

$n$-dimensional vector $\mathbf{q}$ as link-side position. The link-side dynamics of the manipulator can be written as

$$
\mathbf{M}(\mathbf{q}) \ddot{\mathbf{q}}+\mathbf{C}(\mathbf{q}, \dot{\mathbf{q}}) \dot{\mathbf{q}}+\mathbf{g}(\mathbf{q})+\mathbf{V} \dot{\mathbf{q}}+\mathbf{f}=\mathbf{t}+\tau_{\text {ext }}=\tau_{\text {tot }},
$$

where $\mathbf{M}(\mathbf{q})$ is the symmetric positive definite inertia matrix, $\mathbf{C}(\mathbf{q}, \dot{\mathbf{q}})$ is the Coriolis and centrifugal term, $\mathbf{g}(\mathbf{q})$ is the gravity term, and $\mathbf{V} \dot{\mathbf{q}}+\mathbf{f}$ is the link-side friction including viscous term $\mathbf{V}_{n \times n} \dot{\mathbf{q}}$ and Coulomb term $\mathbf{f}$, where $\mathbf{V}_{n \times n}$ is the diagonal matrix of viscous friction coefficient. On the right side of the equation, $\boldsymbol{\tau}_{\text {tot }}$ is the total active torque vector acting on the manipulator including the driving torque term $\tau$ and the external torque term $\boldsymbol{\tau}_{\text {ext }}$.

Most manipulator joints are equipped with harmonic reducer. The reducer magnifies motor torque and motorside inertia, and friction between link and motor side is introduced, as shown in Figure 2. The dynamics of motor side can be described as

$$
\mathbf{J}_{m} \ddot{\boldsymbol{\theta}}+\mathbf{V}_{m} \dot{\boldsymbol{\theta}}+\mathbf{f}_{m}+\mathbf{H}^{-1} \boldsymbol{\tau}=\boldsymbol{\tau}_{m}
$$

where $H$ is an $n \times n$ diagonal matrix of the reduction ratio, $\mathbf{J}_{m}$ represents the inertia of motor rotor and wave generator in harmonic drive, $\tau_{m}$ is the motor torque, $\mathbf{V}_{m} \dot{\boldsymbol{\theta}}+\mathbf{f}_{m}$ is the motor-side friction including viscous and Coulomb terms, $\mathbf{V}_{m}$ is an $n \times n$ diagonal matrix of motor side viscous friction coefficient, and $\theta$ is the motor-side position vector:

$$
\boldsymbol{\theta}=\mathbf{H q} \text {. }
$$

The dynamics considering actuator dynamics can be modeled as follows:

$$
\left[\mathbf{M}(\mathbf{q})+\mathbf{H}^{2} \mathbf{J}_{m}\right] \ddot{\mathbf{q}}+\mathbf{C}(\mathbf{q}, \dot{\mathbf{q}}) \dot{\mathbf{q}}+\mathbf{g}(\mathbf{q})+\left(\mathbf{V}+\mathbf{H}^{2} \mathbf{V}_{m}\right) \dot{\boldsymbol{\theta}}+\left(\mathbf{f}+\mathbf{H} \mathbf{f}_{m}\right)=\mathbf{H} \boldsymbol{\tau}_{m}+\boldsymbol{\tau}_{\mathrm{ext}} .
$$

Remark 1. Reason of considering actuator dynamics. External contact information is transmitted to motor current through the reducer, which adds interference information. The influence of the reducer in active control and back driving is different. In active control, joint motor torque does work to the manipulator and the environment, and the actuator is the motor. At this time, the reduction ratio $\mathbf{H}$ reduces the inertia and viscous friction of the system and plays a positive role in the active control. For back driving, however, the external force does work on the manipulator. The effect of actuator dynamics is amplified when the external force acts as the driving force. As shown in the system dynamics (4), the motor inertia $\mathbf{J}_{m}$ and motor-side viscous friction $\mathbf{V}_{m} \dot{\boldsymbol{\theta}}$ are amplified by squared reduction ratio, and motor-side Coulomb friction $\mathbf{f}_{m}$ is also amplified. The influence is greater when the reduction ration is larger. Therefore, if motor current command is used for estimating external torque, then the influence of actuator dynamics cannot be ignored, especially for the manipulator with high reduction ratio.

2.2.2. Momentum Observer Based on Current Command. The generalized momentum of the manipulator is defined as

$$
\mathbf{p}(t)=\mathbf{M}(\mathbf{q}) \dot{\mathbf{q}} .
$$

Here, we define the $n$-dimensional system inertia matrix as 


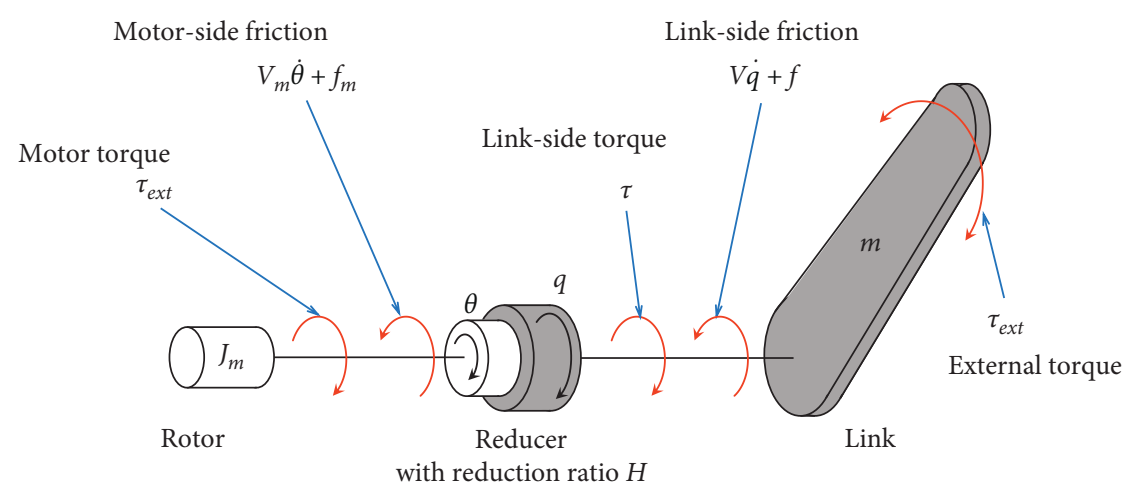

Link side

Motor side

Figure 2: Robot joint with harmonic reducer.

$$
\mathbf{B}(\mathbf{q})=\mathbf{M}(\mathbf{q})+\mathbf{H}^{2} \mathbf{J}_{m} .
$$

The generalized momentum involved total system inertia comes to

$$
\mathbf{p}_{B}(t)=\mathbf{B}(\mathbf{q}) \dot{\mathbf{q}}
$$

The motor torque $\tau_{m}$ is associated with motor current; that is,

$$
\boldsymbol{\tau}_{m}=\mathbf{k}_{\tau} \mathbf{i}_{m}
$$

where $\mathbf{k}_{\tau}$ is an $n \times n$ diagonal matrix of motor torque constant and $\mathbf{i}_{m}$ indicates the motor current. However, the motor current is usually difficult to measure and filter. Fortunately, the performance of current loop of commercial drivers can be sufficiently accurate that the actual motor current can be replaced by the motor torque command generated by the servo algorithm. That is,

$$
\mathbf{i}_{m} \cong \mathbf{c}_{A} \mathbf{i}_{c}
$$

where $\mathbf{i}_{c}$ is the current command vector given by the controller and $\mathbf{c}_{A}$ is an $n \times n$ diagonal matrix of the scale factor between the controller and the servo driver. The current command is often mixed with considerable noise. Therefore, real-time filtered current command $\widehat{\mathbf{i}}_{c}$ is used. From the above, the motor torque is obtained by

$$
\boldsymbol{\tau}_{m}=\mathbf{k}_{\tau} \mathbf{c}_{A} \hat{\mathbf{i}}_{c} .
$$

The total friction term is represented by

$$
\boldsymbol{\tau}_{t f}=\left(\mathbf{V}+\mathbf{H}^{2} \mathbf{V}_{m}\right) \dot{\boldsymbol{\theta}}+\left(\mathbf{f}+\mathbf{H} \mathbf{f}_{m}\right)
$$

The $n$-dimensional residual vector $\hat{r}$ of momentum observer defined in previous research $[14,15]$ is modified as

$\widehat{\mathbf{r}}(t)=\mathbf{K}_{I}\left[\Delta \mathbf{p}_{B}-\int\left(\mathbf{H k}_{\tau} \mathbf{c}_{A} \widehat{\mathbf{i}}_{c}+\mathbf{C}^{T}\left(\mathbf{q}_{\mathbf{a}}, \dot{\mathbf{q}}_{a}\right) \dot{\mathbf{q}}_{a}-\mathbf{g}\left(\mathbf{q}_{\mathbf{a}}\right)-\boldsymbol{\tau}_{\mathrm{tf}}+\widehat{\mathbf{r}}\right) \mathrm{d} t\right]$,

where $\Delta \mathbf{p}_{B}=\mathbf{p}_{B}(t)-\mathbf{p}_{B}(0)$, and $\mathbf{K}_{I}$ is a diagonal gain matrix. The momentum observer (12) only needs motor-side information including actual joint position $\mathbf{q}_{\mathbf{a}}$, joint velocity $\dot{q}_{a}$, and current command $\widehat{\mathbf{i}}_{c}$. Only a basic motor position feedback is actually required; no more force/torque sensors are needed. According to the skew symmetry of $\mathbf{M}\left(\mathbf{q}_{\mathbf{a}}\right)-\mathbf{C}\left(\mathbf{q}_{\mathbf{a}}, \dot{\mathbf{q}}_{\mathbf{a}}\right)$, we can derive that

$$
\dot{\mathbf{M}}\left(\mathbf{q}_{\mathrm{a}}\right)=\mathbf{C}\left(\mathbf{q}_{\mathrm{a}}, \dot{\mathbf{q}}_{\mathrm{a}}\right)+\mathbf{C}^{T}\left(\mathbf{q}_{\mathrm{a}}, \dot{\mathbf{q}}_{\mathbf{a}}\right) \text {. }
$$

Using (4)-(6) and (13), the derivative of $\mathbf{p}_{B}(t)$ is

$$
\dot{\mathbf{p}}_{B}=\mathbf{C}^{T}\left(\mathbf{q}_{\mathbf{a}}, \dot{\mathbf{q}}_{\mathbf{a}}\right) \dot{\mathbf{q}}_{\mathbf{a}}+\mathbf{H} \tau_{m}+\boldsymbol{\tau}_{\mathrm{ext}}-\mathbf{g}\left(\mathbf{q}_{\mathbf{a}}\right)-\boldsymbol{\tau}_{t f} .
$$

With (14), the dynamics of $\widehat{\mathbf{r}}$ is

$$
\dot{\widehat{\mathbf{r}}}=\mathbf{K}_{I} \boldsymbol{\tau}_{\text {ext }}-\mathbf{K}_{I} \widehat{r} \text {. }
$$

The Laplace transformation of (15) for each joint is

$$
\widehat{r}^{i}=\frac{K_{I}^{i}}{s+K_{I}^{i}} \tau_{\text {ext }}^{i}, \quad i \in\{1, \ldots, n\} .
$$

Therefore, the vector $\widehat{\mathbf{r}}$ is essentially a first-order filtering of external disturbance on each joint. When the gain $\mathbf{K}_{I}$ is as large as possible, the residual approaches the external torque as

$$
\widehat{\mathbf{r}} \approx \tau_{\mathrm{ext}} \Rightarrow \widehat{\boldsymbol{\tau}}_{\mathrm{ext}}=\widehat{\mathbf{r}}
$$

For manipulators equipped with joint-side torque sensors, the actuator dynamics need not be considered given that external information is directly detected by the sensors. With direct access to link side torque $\tau$, the momentum observer becomes

$$
\widehat{r}=\mathbf{K}_{I}\left[\Delta \mathbf{p}-\int\left(\boldsymbol{\tau}+\mathbf{C}^{T}\left(\mathbf{q}_{\mathbf{a}}, \dot{\mathbf{q}}_{\mathbf{a}}\right) \dot{\mathbf{q}}_{\mathbf{a}}-\mathbf{g}\left(\mathbf{q}_{\mathbf{a}}\right)+\widehat{r}\right) \mathrm{d} t\right] .
$$

This method involving torque sensors is also suitable for our control framework. However, it is used in our experiments as the comparison with sensorless method to evaluate the performance of sensorless control strategy.

\subsection{Design of Compliance Control Algorithm}

2.3.1. Methodology. The three haptic features essentially abstract three physical models into control effect. For a 
rotatory joint, the haptic features become the torsional spring with stiffness $k$, the rotational damper $d$, and the moment of inertia $J$. The physical properties are

$$
\begin{aligned}
\tau & =k \Delta q, \\
\tau & =\mathrm{d} \dot{q}, \\
\tau & =J \ddot{q} .
\end{aligned}
$$

Inspired by equation (19), three control algorithms map the joint external torque onto desired joint position, joint velocity, and joint acceleration to generate reference trajectory, as shown in Figure 1. The mapping algorithms can be written as

$$
\begin{aligned}
\Delta q_{d} & =P(s) \widehat{\tau}_{\mathrm{ext}}, \\
\dot{q}_{d} & =Q(s) \widehat{\tau}_{\mathrm{ext}}, \\
q_{d} & =R(s) \widehat{\tau}_{\mathrm{ext}},
\end{aligned}
$$

where $P(s), Q(s), R(s)$ are three filters called haptic filters. $\widehat{\tau}_{\text {ext }}$ is the observed external torque, and $\Delta q_{d}, \dot{q}_{d}, \ddot{q}_{d}$ are the desired trajectory characteristics.

Position servo control system of manipulators has been well developed today. Most of the commercial drivers can conduct accurate real-time trajectory tracking. At this point, we assume that the low-level position controller of the manipulator has a perfect performance which leads to a negligible error of trajectory tracking. Thus, the actual trajectory is equivalent to the reference trajectory. That is,

$$
\begin{aligned}
& q_{a} \cong q_{r}, \\
& \dot{q}_{a} \cong \dot{q}_{r}, \\
& \ddot{q}_{a} \cong \ddot{q}_{r},
\end{aligned}
$$

where $q_{a}, \dot{q}_{a}, \ddot{q}_{a}$ are the actual trajectories.

The external disturbance torque is obtained by the momentum observer in Section 2.2. On the basis of former research [40], we assume the observing error is negligible. The observed disturbance torque can be considered the real disturbance torque. That is,

$$
\widehat{\tau}_{\text {ext }} \cong \tau_{\text {ext }},
$$

where $\tau_{\text {ext }}$ represents the real disturbance torque arising from external contacts. Therefore, the mapping algorithm comes to

$$
\begin{aligned}
\Delta q_{d} & =P(s) \tau_{\text {ext }}, \\
\dot{q}_{d} & =Q(s) \tau_{\text {ext }}, \\
\ddot{q}_{d} & =R(s) \tau_{\text {ext }} .
\end{aligned}
$$

Therefore, the haptic filters represent the relationship between actual disturbance and trajectory.

In physical models, the three characteristics are constant; if the amplitude and phase frequency characteristics of $P(s), Q(s), R(s)$ are close to those of constants, then $P(s), Q(s), R(s)$ could be viewed as stiffness, damping, and inertia. Then, we can define $P(s), Q(s), R(s)$ as virtual rotary stiffness, damping, and inertia of robot joint, which determine the relationships between applied disturbance torque and joint angular displacement, velocity, or acceleration, respectively.

2.3.2. Haptic Filter. The observed external torques are coupled with considerable noise, especially when motor current is used as the signal input. Direct mapping with a gain will lead to vibration and discontinuity of trajectory. Therefore, a haptic filter is introduced to filter the noise and enhance the interaction experience. For uniformity and simplicity of the control algorithm, three haptic filters have the same structure as follows:

$$
\left\{\begin{array}{l}
P(s)=K_{P} \frac{p_{P} \omega_{n P}^{2}}{\left(s+p_{P}\right)\left(s^{2}+2 \zeta_{P} \omega_{n P} s+\omega_{n P}^{2}\right)}=K_{P} F_{P}(s), \\
Q(s)=K_{Q} \frac{p_{Q} \omega_{n Q}^{2}}{\left(s+p_{Q}\right)\left(s^{2}+2 \zeta_{Q} \omega_{n Q} s+\omega_{n Q}^{2}\right)}=K_{Q} F_{Q}(s), \\
R(s)=K_{R} \frac{p_{R} \omega_{n R}^{2}}{\left(s+p_{R}\right)\left(s^{2}+2 \zeta_{R} \omega_{n R} s+\omega_{n R}^{2}\right)}=K_{R} F_{R}(s),
\end{array}\right.
$$

where $K_{P}, K_{Q}, K_{R}$ are scale factors. Applying such structure makes the generated trajectory present third-order derivative characteristic. Thus, for the worst cases, the position, velocity, and acceleration commands are all smooth curves. Accordingly, acceleration discontinuity will be eliminated. The haptic filter is essentially a low-pass filter. Appropriate bandwidth will help eliminate the noise of the observed disturbance torque $\widehat{\tau}_{\text {ext }}$. The convergence speed of compliance controller refers to the respond speed of compliant behavior to the external torque. Given that the low-level position controller can achieve natural high bandwidth, the bandwidth of haptic filter determines the convergence speed of the desired trajectory command. Thus, the cutoff frequency of the filter (24) should be set to an appropriate value to balance the filtering and the response speed. A higher bandwidth will lead to a faster convergence speed but will bring more noise from the external torque signals.

2.4. Compliance Control Law. Here, the single-joint control methodology is extended to an $n$-DOF robot manipulator control. We define $n \times n$ diagonal matrix of haptic filters $\mathbf{P}, \mathbf{Q}, \mathbf{R}$ for three haptic features as

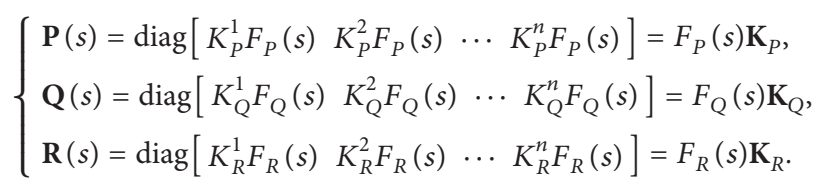

The parameters of $F_{P}(s), F_{Q}(s), F_{R}(s)$ are consistent for each joint in one control method. $\mathbf{K}_{P}, \mathbf{K}_{Q}, \mathbf{K}_{R}$ are diagonal matrices of scale factors, and their components are 
adjustable for each joint. By combining the haptic filter matrix with the mapping algorithm, we have

$$
\left\{\begin{array}{l}
\mathbf{q}_{d}=P \widehat{\boldsymbol{\tau}}_{\mathrm{ext}}=F_{P}(s) \mathbf{K}_{P} \widehat{\boldsymbol{\tau}}_{\mathrm{ext}}, \\
\dot{\mathbf{q}}_{d}=Q \widehat{\boldsymbol{\tau}}_{\mathrm{ext}}=F_{Q}(s) \mathbf{K}_{Q} \widehat{\boldsymbol{\tau}}_{\mathrm{ext}}, \\
\ddot{\mathbf{q}}_{d}=R \widehat{\boldsymbol{\tau}}_{\mathrm{ext}}=F_{R}(s) \mathbf{K}_{R} \widehat{\boldsymbol{\tau}}_{\mathrm{ext}},
\end{array}\right.
$$

where $\mathbf{q}_{d}, \dot{\mathbf{q}}_{d}, \ddot{\mathbf{q}}_{d}$ represent $n$-dimensional desired joint space position, velocity, and acceleration vectors. The desired trajectory characteristics $\mathbf{q}_{d}, \dot{\mathbf{q}}_{d}, \ddot{\mathbf{q}}_{d}$ are used to generate the reference trajectory. For each joint of manipulator, we have

$$
\left\{\begin{array}{l}
\left|\widehat{\tau}_{\text {ext }}^{i}\right|=\frac{1}{K_{P}^{i}} \frac{1}{|F(j \omega)|}\left|q_{d}^{i}\right| \Leftrightarrow \tau=k \Delta q, \\
\left|\widehat{\tau}_{\text {ext }}^{i}\right|=\frac{1}{K_{Q}^{i}} \frac{1}{|F(j \omega)|}\left|\dot{q}_{d}^{i}\right| \Leftrightarrow \tau=\mathrm{d} \dot{q}, \quad i \in\{1, \ldots, n\}, \\
\left|\widehat{\tau}_{\text {ext }}^{i}\right|=\frac{1}{K_{R}^{i}} \frac{1}{|F(j \omega)|}\left|\ddot{q}_{d}^{i}\right| \Leftrightarrow \tau=J \ddot{q} .
\end{array}\right.
$$

According to equation (27), the trajectory characteristics of the $i$ th joint $\left|q_{d}^{i}\right|,\left|\dot{q}_{d}^{i}\right|,\left|\ddot{q}_{d}^{i}\right|$ are proportional to the $i$ th external torque $\left|\hat{\tau}_{\text {ext }}^{i}\right|$. Therefore, $1 / K_{P}^{i}|F(j \omega)|$ indicates the virtual joint stiffness $k$ of the $i$ th joint, $1 / K_{Q}^{i}|F(j \omega)|$ is the virtual joint damping $d$, and $1 / K_{R}^{i}|F(j \omega)|$ represents the virtual joint inertia $J$. As the parameters of $F(s)$ are set, the virtual joint haptic features can be linearly tuned by adjusting scale factors $1 / K_{P}^{i}, 1 / K_{Q}^{i}, 1 / K_{R}^{i}$, which are independent between each joint.

Remark 2. The independent adjustability of joint space characteristics can make the adjustment of haptic features more flexible. The virtual characteristics can be distributed to the joints in different values. For example, the virtual stiffness values of joints near robot base are set larger than those of the other joints. This way makes the base of robot arm harder to push, but much softer near the end-effector. Other configurations can also be set depending on the users.

The reference trajectory $\mathbf{q}_{r}$ of three control schemes is generated by the desired trajectory characteristics $\mathbf{q}_{d}, \dot{\mathbf{q}}_{d}, \ddot{\mathbf{q}}_{d}$ as follows:

$$
\left\{\begin{array}{l}
\mathbf{q}_{r}=\mathbf{q}_{d}=F(s) \mathbf{K}_{P} \widehat{\boldsymbol{\tau}}_{\mathrm{ext}} \\
\mathbf{q}_{r}=\frac{1}{s^{2}} \dot{\mathbf{q}}_{d}=\frac{1}{s^{2}} F(s) \mathbf{K}_{R} \widehat{\boldsymbol{\tau}}_{\mathrm{ext}} \\
\mathbf{q}_{r}=\frac{1}{s^{2}} \ddot{\mathbf{q}}_{d}=\frac{1}{s^{2}} F(s) \mathbf{K}_{R} \widehat{\boldsymbol{\tau}}_{\mathrm{ext}}
\end{array}\right.
$$

The observed external torque $\widehat{\tau}_{\text {ext }}$ is devised by the momentum observer discussed in the following section.
Remark 3. In stiffness control mode, the rigid manipulator joint becomes elastic joint, and the joint elasticity is configurable. This mode is suitable for variable stiffness control for the manipulator with rigid joints. Damping control method makes the joint velocity proportional to joint external torque, which can be applied to physical hands-on driving tasks. Inertia control adjusts joint acceleration proportionally to external force. In this control mode, the manipulator joint will keep moving in constant velocity when no external disturbance exists. When external contact occurs, the robot arm will accelerate in the same direction with external force. This control scheme can be used for dynamics simulation in aerospace such as spacecraft docking operation.

\section{Simulation of Control Strategy}

The control strategy is prevalidated by simulation before conducting any experiments. A 2-DOF manipulator model is built, and its parameters are set as in Figure 3. The control system is built based on the control strategy in Figure 1. During the simulation, an external force $F$ will act along $F_{\text {ext } 2}$ and then along $F_{\text {ext } 1}$. The results of the three control methods are presented in Figure 4. The observed external torque and joint trajectory curves of the two joints are plotted.

The parameters of stiffness feature filter $P(s)$ are set as follows: $K_{P}=0.0035, p_{1}=7000 \pi, \omega_{n}=2000 \pi$, and $\zeta=0.6$. The gain of momentum observer $K_{I}=500$. External force $F=150$ N. As shown in Figure 4(a), when the manipulator first interacts with $F_{\text {ext } 2}$ acting on link 2 during $0.5-2.5 \mathrm{~s}$, two joints detect external torque, and joint positions react to the external torque proportionally. When the manipulator is interacting with $F_{\text {ext } 1}$ acting on link 1 from $3 \mathrm{~s}$ to $5 \mathrm{~s}$, only joint $J_{1}$ detects external torque, and only joint $J_{1}$ reacts. The joint positions react proportionally to joint space external torque. The manipulator presents spring feature and sensitivity to the external force acting on the whole arm.

The parameters of damping feature filter $Q(s)$ are set differently as follows: $K_{Q}=0.1, p_{1}=1000 \pi, \omega_{n}=1000 \pi$, and $\zeta=0.9$. The gain of momentum observer $K_{I}=500$. External force $F=10 \mathrm{~N}$. The simulation result is illustrated in Figure 4(b). When the manipulator interacts with external force, the joint position is nearly a ramp function with a constant derivative. This condition means the joint velocity is proportional to joint space external torque. The manipulator shows damper feature and global sensitivity.

The parameters of inertia feature filter $R(s)$ are $K_{R}=0.003, p_{1}=1000 \pi, \omega_{n}=1000 \pi$, and $\zeta=0.9$. The gain of momentum observer $K_{I}=500$. External torque $F=10 \mathrm{~N}$. Simulation results are shown in Figure 4(c). When external force acts on the manipulator, the joint velocity is a ramp function and the joint position is quadratic curve, which means the joint acceleration is a constant. As shown in the figure of joint 1 (the upper figure of Figure 4(c)), the slope of joint velocity curve is unsurprisingly proportional to observed external joint torque. Therefore, the joint acceleration is nearly proportional to external torque. 


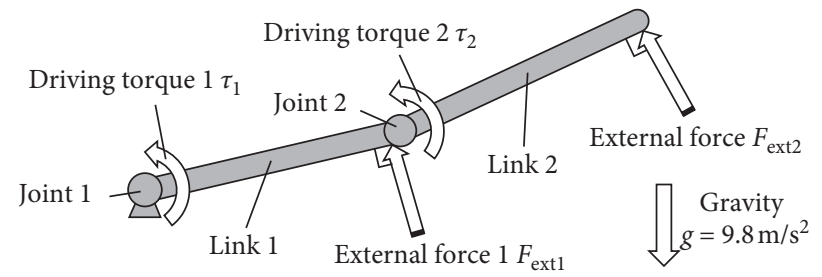

FIGURE 3: 2-DOF simulation model. Length of each link is $0.3 \mathrm{~m}$, mass of each link is $0.73 \mathrm{~kg}$, driving torque $\tau_{1}$, $\tau_{2}$ are applied on joint $J_{1}$ and $J_{2}$, and external force $F_{\text {ext } 1}, F_{\text {ext } 2}$ are applied to links 1 and 2 .
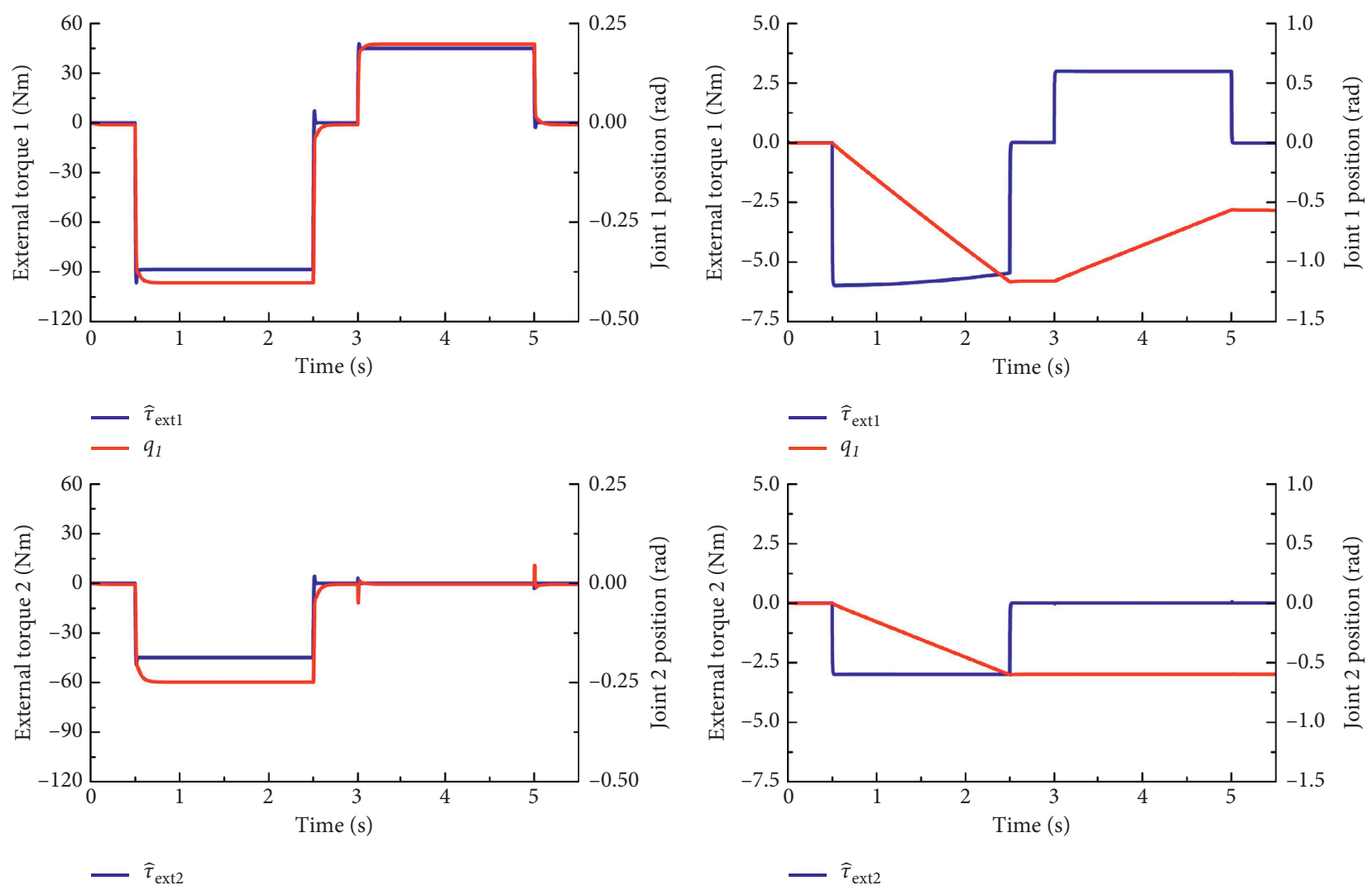

(a)

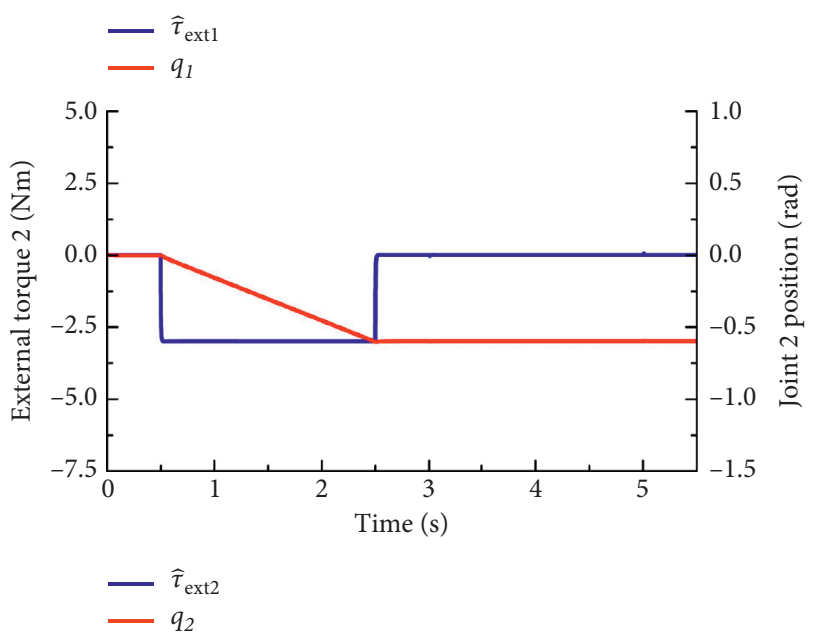

(b)

FIgURE 4: Continued. 

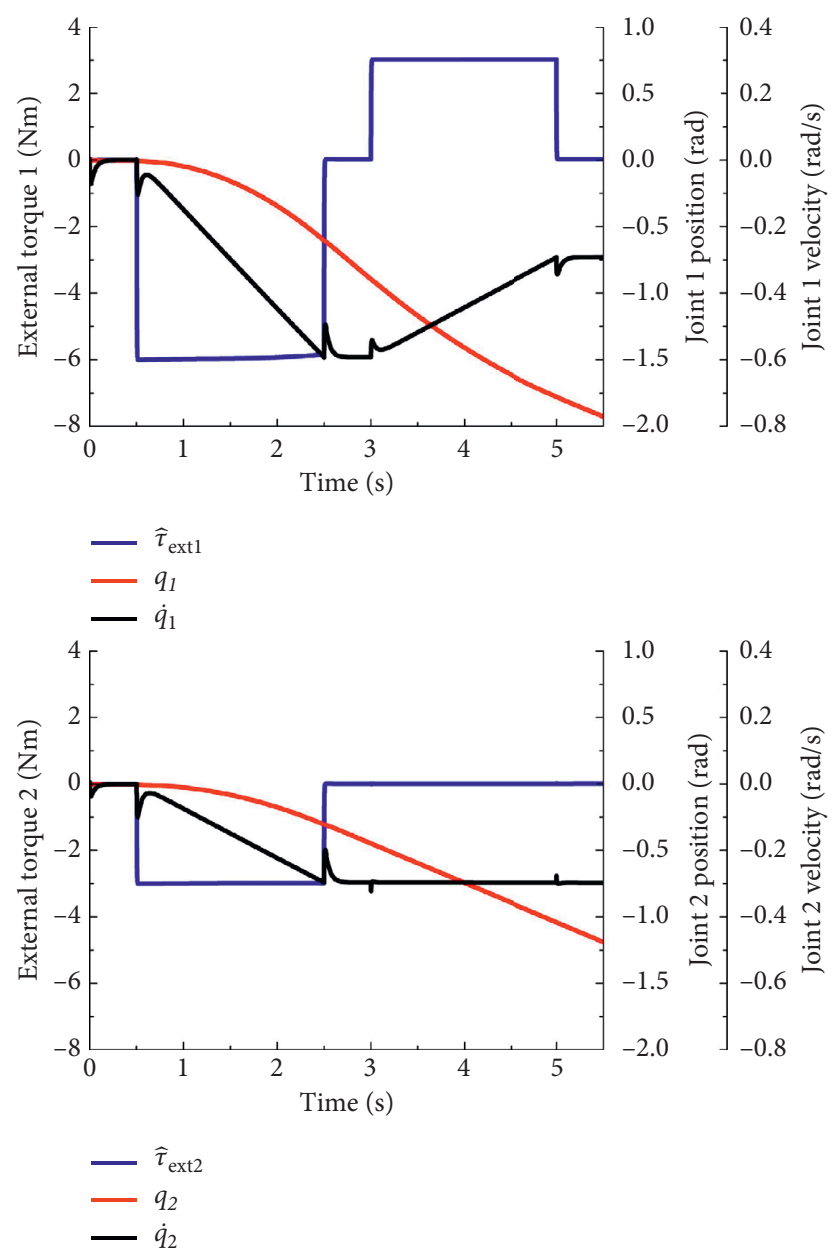

(c)

Figure 4: Simulation results of three control methods on 2-DOF robot structure. (a) Stiffness control, $F=150$ N. (b) Damping control, $F=10 \mathrm{~N}$. (c) Inertia control, $F=10 \mathrm{~N}$. The simulation results show that the compliance control strategy is effective.

\section{Experiments}

This part took a 2-DOF robot as an example to verify the effectiveness of the control strategy. First, the design of the experimental platform was introduced. Then, the performance of the modified momentum observer was verified. The three control schemes would be validated by comparison experiments using sensor-based and sensorless momentum observers.

4.1. Development of Robot Experiment Platform. As shown in Figure 5, the 2-DOF experiment platform consisted of two typical robot joints composed of BLDC motor and harmonic reducer. The joint position was acquired by motor-side highresolution incremental encoder and reduction ratio. Meanwhile, joint torque sensors were also equipped on the link side to measure actual torque for comparison experiments. The control system used a widely used commercial controller and servo drives to ensure control performance.

The dynamics of the experiment platform was first built using CAD and components parameters from the manufacturers. The friction compensation was conducted as follows. First, motor currents were recorded when the joints move at a group of constant joint velocity. The motor-side torque was obtained according to equation (8) and then amplified into the driving torque by the deceleration ratio. The measured actual torque was subtracted from driving torque to obtain friction term. By quadratic fitting the difference values under different velocities, the fitting curves of joint positions were obtained and finally applied to the dynamic model. The low-level position controller was executed every $0.5 \mathrm{~ms}$. The high-level control loop was executed every $4 \mathrm{~ms}$. The refresh period of the momentum observer was $1.22 \mathrm{~ms}$.

4.2. Validation of Sensorless Momentum Observer. In this part, the effectiveness of the observer was verified by comparing the estimated external torque with the actual external torque measured by the torque sensors. Both momentum observers based on torque sensors and current command were validated. As shown in Figure 6(a), the manipulator moved along a set trajectory under position control. We defined the horizontal posture as the initial 


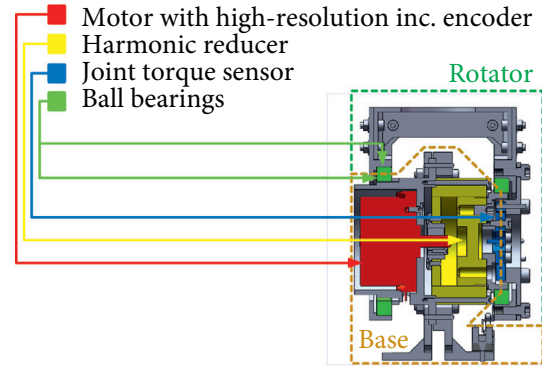

(a)

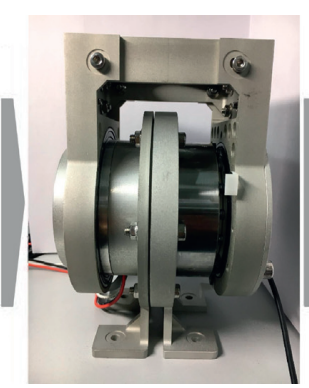

(b)

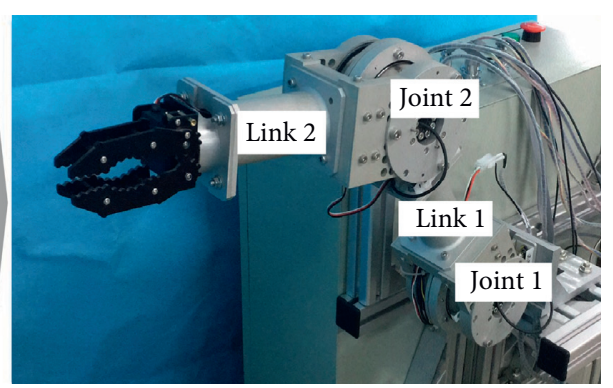

(c)

Figure 5: Experimental platform. (a) Sectional view of a single robot joint and selected components. (b) Single joint of the platform and its components. (c) 2-DOF rigid manipulator experiment platform equipped with high-resolution motor encoders and joint torque sensors.
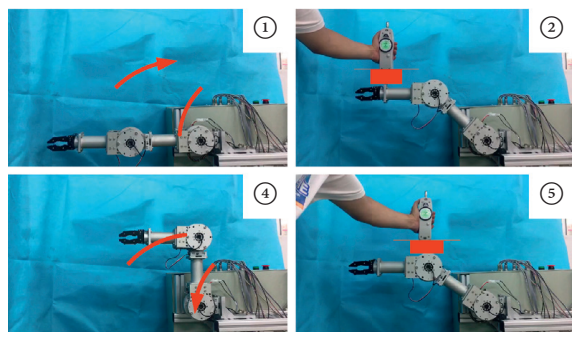

(a)
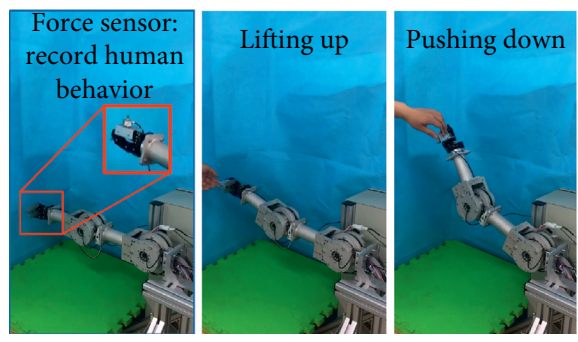

(b)

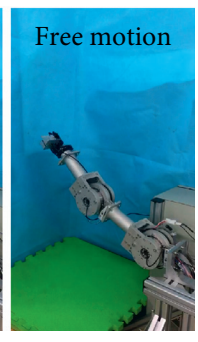

Figure 6: Experimental process for validation of (a) momentum observer in Section 4.2 and (b) compliance control in Section 4.4. A force sensor is equipped to record human behavior. Lifting and pushing forces are positive and negative, respectively.

point, that is, $\left(q_{1}, q_{2}\right)^{T}=(0,0)^{T}$, and upward movement was defined as positive direction. The manipulator reciprocated along a trajectory from $(0,0)^{T}$ to $(\pi / 2,-\pi / 2)^{T}$, with a velocity of $5.8^{\circ} / \mathrm{s}$ for each joint. The gain for each joint was set as $K_{I}^{1}=K_{I}^{2}=100$. During the motion, the external force was applied to the upper and lower points of the two links. The actual external torque used for comparison was measured by the torque sensor as follows. The joint torque with external contact and without external torque was recorded separately in two motion cycles. The difference between the two was the actual external torque.

The comparison results are presented in Figure 7. For momentum observer based on joint torque sensor (18), the noise of joints 1 and 2 without external force were within 1 and $0.5 \mathrm{Nm}$, respectively. When external force was applied, the estimation errors of external torque were both close to $0.3 \mathrm{Nm}$ for two joints. Biases were observed in Figures 7(a) and $7(\mathrm{~b})$, and they were mainly caused by modeling errors. For the momentum observer based on current command (12), the tracking errors of joints 1 and 2 were within 2 and $0.5 \mathrm{Nm}$, respectively, when no contact on the robot was considered. With external contacts, the error of observer was approximately $20 \%$ of the actual external torque of joint 1 and $0.5 \mathrm{Nm}$ of joint 2 . Here, the error was mostly caused by electrical noise and the modeling errors.

Remark 4. The results show that both methods responded rapidly to external disturbance. The accuracy of the sensorless momentum observer was a bit lower than that of the sensor-based method but was still sufficiently accurate for external torque estimation. The tracking error most likely caused by the modeling uncertainty was within an acceptable range. In general, the momentum observer based on current command was proven to be effective. A small sacrifice of estimation accuracy brought the benefit of not relying on force/torque sensors.

A peak of observed external torque of joint 2 was found at the moment of changing motion direction (Figure 6(a)). The reason is the inaccuracy of the joint friction modeling at the static point, namely, the stiction. In fact, the stiction is related to many uncertain factors, such as robot posture, temperature, and hysteresis. Accurate modeling is very difficult, which is beyond the scope of this research.

\subsection{Limitation of Applying Sensorless Momentum Observer to} Torque Servo. During our early research, reflex torque reaction control [15] was investigated using the sensorless momentum observer, and a defect was found in control effects. This method maps estimated external torque obtained by momentum observer on joint torque command with gravity compensation, and the control law is

$$
\boldsymbol{\tau}_{\text {command }}=\overline{\mathbf{g}}(\mathbf{q})+\mathbf{K}_{R} \widehat{r},
$$

where $\bar{g}$ is the compensation of gravity term and $\mathbf{K}_{R}$ is the scale factor diagonal matrix. In the experiment of [15], link side torque sensor signal was assumed as commanded torque, and good control effect was achieved.

To achieve sensorless control, we reproduced the experiments of reflex torque control strategy, and both sensorand current-based momentum observers were utilized. As presented in Figure 8, collision detection was triggered as 


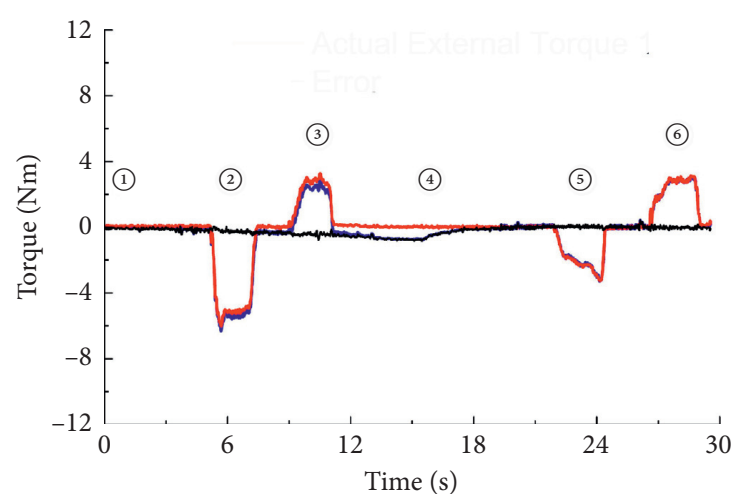

- Observed external torque 1

- Actual external torque 1

— Error

(a)

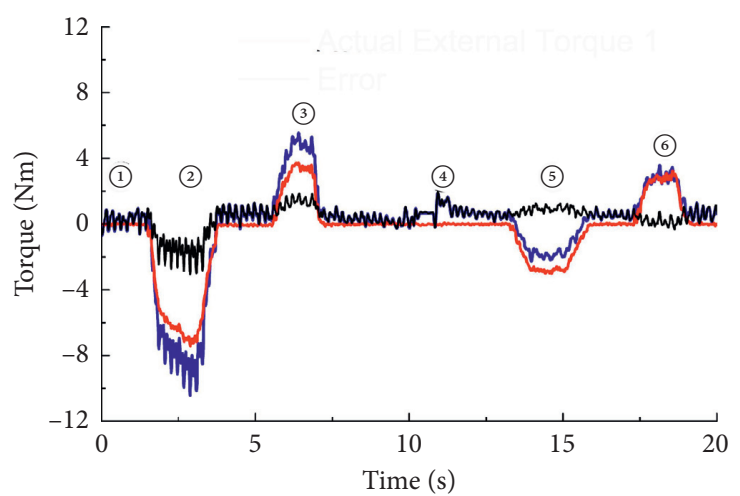

Observed external torque 1
_ Actual external torque 1
Error

(c)

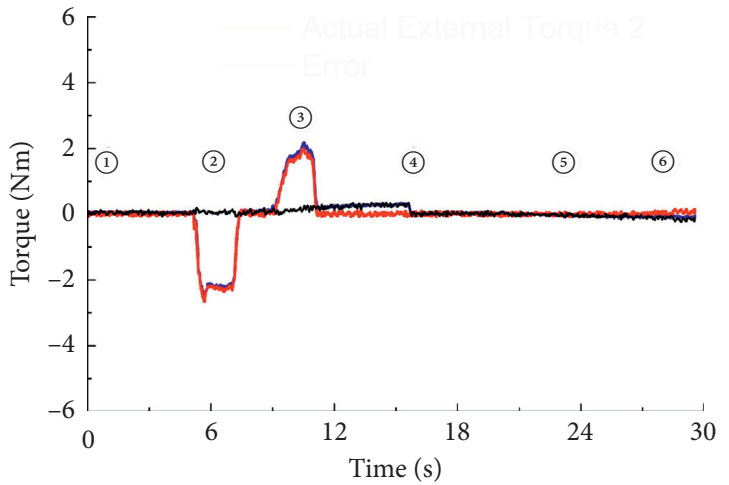

- Observed external torque 2

- Actual external torque 2

_ Error

(b)

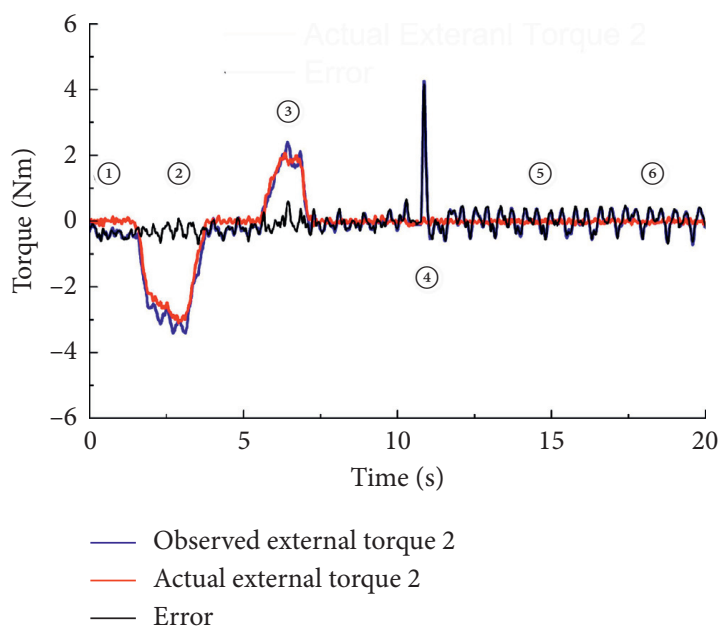

(d)

Figure 7: Performance of momentum observer using the torque sensor ((a), (b)) and the current command ((c), (d)) on two joints. The numbers 1 to 6 in the figure correspond to the moments in Figure 6(a).

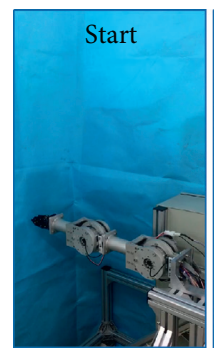

(a)

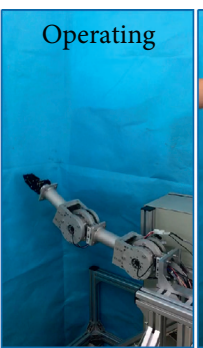

(b)

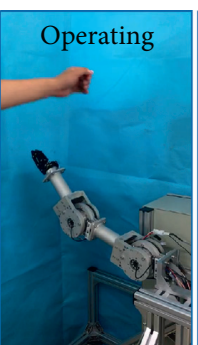

(c)

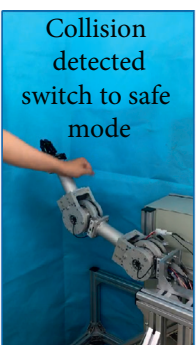

(d)

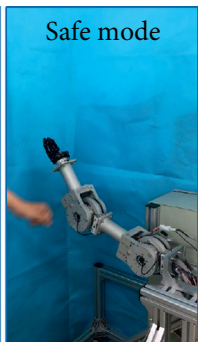

(e)

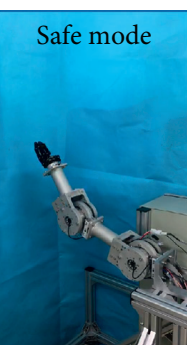

(f)

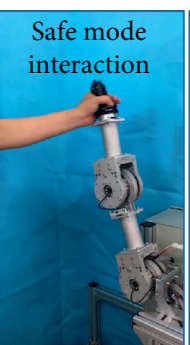

(g)

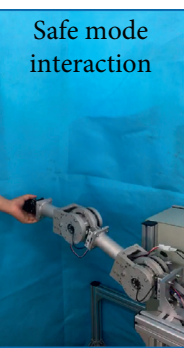

(h)

Figure 8: Collision detection and physical human-robot interaction using reflex torque reaction control.

soon as one of the estimated joint external torque exceeds the detection threshold. Then, operation mode was immediately switched to safe mode, namely, reflex torque reaction strategy. Then, a human-robot interaction experiment in safe mode was conducted.

The detection threshold of the sensor-based momentum observer was set to $(2.5 \mathrm{Nm}, 0.8 \mathrm{Nm})$ which is $(6.83 \%, 5.64 \%)$ of the maximum output of joints 1 and 2 . A higher threshold
$(4.5 \mathrm{Nm}, 2 \mathrm{Nm})$, i.e., $(12.30 \%, 14.09 \%)$ of the maximum output, was set to cover the noise of the current-based observer. From the results in Figure 9, both methods successfully triggered collision detection when external contact occurred. The detection threshold of the sensor-based momentum observer on LWR-III was set to $10 \%$ of $\max$ joint output [15]. Although the proposed current-based momentum observer needed slightly higher thresholds for 

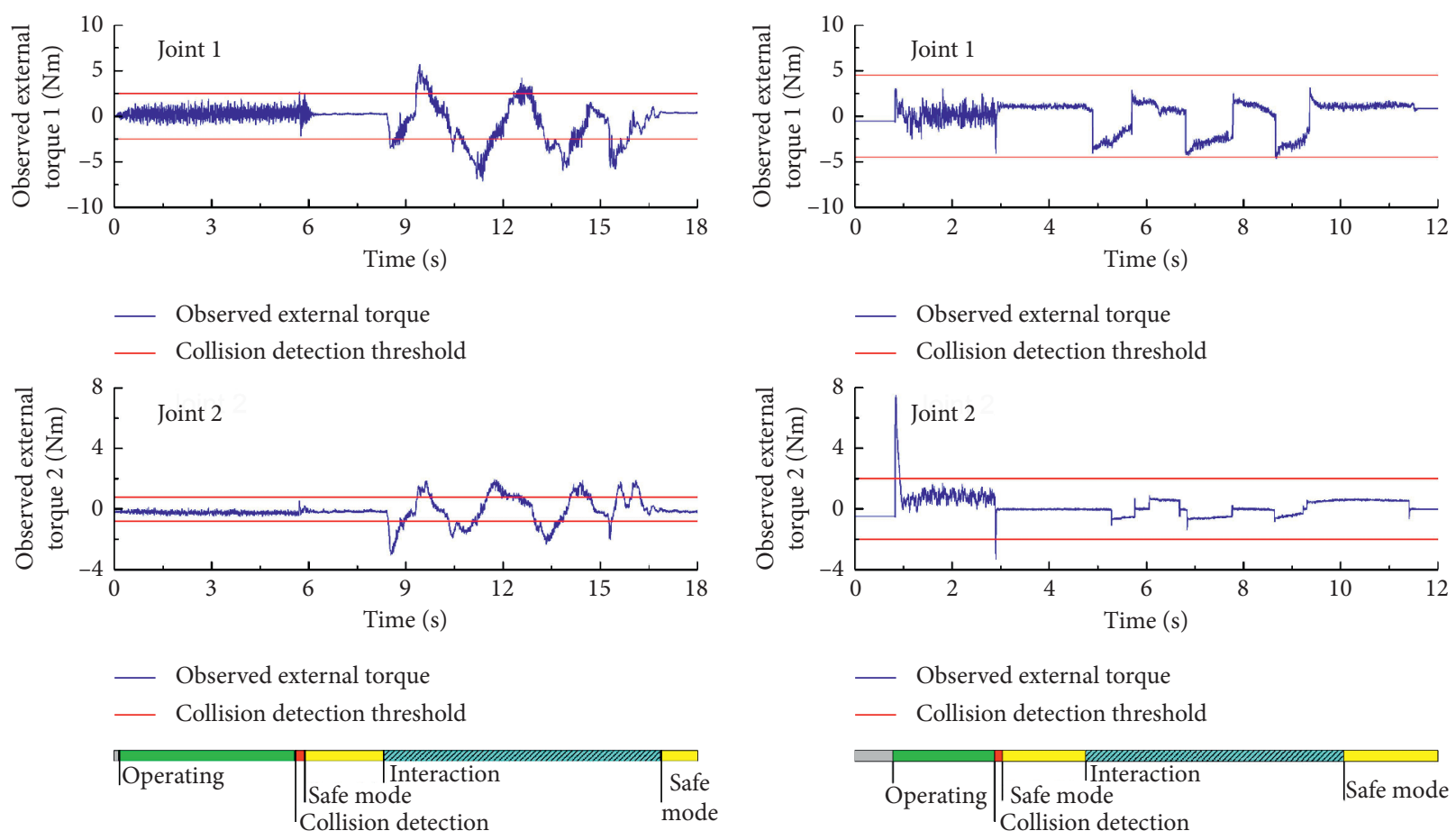

(a)

(b)

Figure 9: Results of reflex torque reaction. (a) Sensor-based momentum observer. (b) Sensorless momentum observer.

collision detection, the dependence of torque sensors was eliminated.

The physical human-robot interaction experiment was conducted after collision detection. The result in Figure 9(a) shows that the operator experienced a smooth interaction using the sensor-based observer. As shown in Figure 9(b), the estimated external torque jumped as the motion direction changed when the current-based observer was applied. This condition indicates that the operator felt discontinuous haptic feeling during the interaction. The reason is the active motor torque and stiction were balanced near the zero-velocity point when the robot was in current control mode. The stiction is greater than sliding friction. Thus, the operator needed to overcome greater friction to transmit the interaction force to the motor side [41]. Therefore, for sensorless momentum observer, directly reflecting the estimated torque on joint torque command is not a proper strategy for physical human-robot interaction. This shortcoming can be alleviated through our proposed algorithm based on the idea of admittance control, which is discussed in Section 4.4.

4.4. Validation of Compliance Control Strategy. Compliance control methods were validated using sensorbased observer (18) and current-based observer (12). A force sensor was equipped at the end of the manipulator to record human behavior and external force. As shown in Figure 6(b), the compliant behavior was triggered, and observed joint torque and joint trajectory were recorded by pushing and pulling the force sensor.
The parameters of the haptic filters were chosen as $p_{P}=p_{Q}=p_{R}=1000 \pi, \quad \omega_{n P}=\omega_{n Q}=\omega_{n R}=1000 \pi, \quad$ and $\zeta_{P}=\zeta_{Q}=\zeta_{R}=0.9$. For stiffness control in Figures 10(a) and $10(\mathrm{~d})$, joint scale factors were set as $K_{P}^{1}=K_{P}^{2}=1.0$; for damping control in Figures 10(b) and 10(e), the scale factors were chosen as $K_{Q}^{1}=K_{Q}^{2}=1.0$; and for inertia control in Figures $10(\mathrm{c})$ and $10(\mathrm{f})$, the scale factors were set as $K_{R}^{1}=K_{R}^{2}=0.3$. The parameters of the momentum observer were set as before. As shown in Figure 10, the actual joint trajectory including joint position, velocity, and acceleration followed joint space external torque obtained by sensor-based momentum observer. This result indicates that the compliance control strategy is effective in sensor-based and sensorless way. The filter caused a slight hysteresis, but it did not affect the overall operating experience. Using the stiffness control method, the joint exhibited a torsion spring effect. The position responded proportionally to estimated joint external torque. For the damping control, joint velocity was positively correlated to estimated joint external torque, like a rotary damper. For the inertia control, the joint position was generated from acceleration which is proportional to estimated external torque. The manipulator felt similar to a floating mass in a zero-gravity environment. Although the sensorless observer had higher noise than the sensor-based observer, the performance of compliant behavior was not affected. The trajectory shows that the compliance control is very smooth.

A small hysteresis was introduced due to the haptic filter. We can imagine the third-order haptic filter as a soft body sandwiched between the human hand and the robot body, which acts as a buffer for external contact. The buffer gives the operator a smoother haptic perceptron during operation 


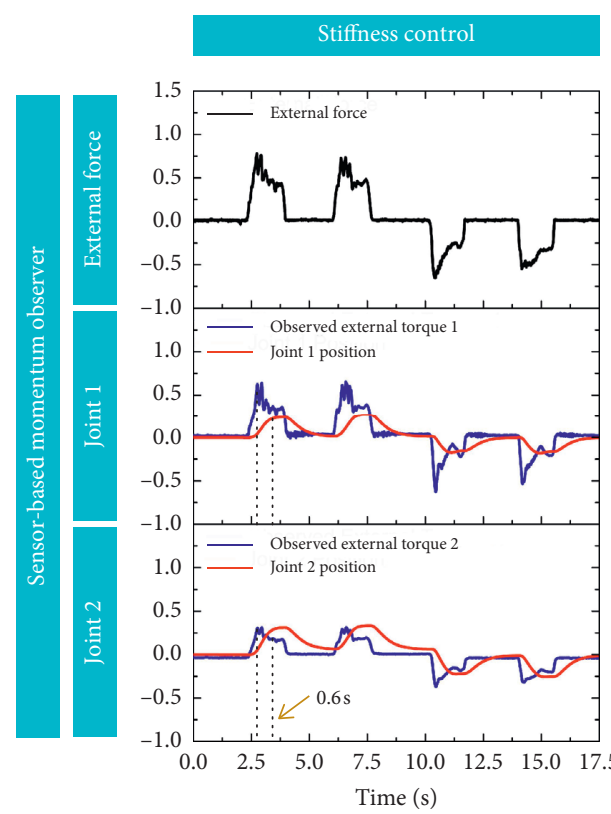

(a)

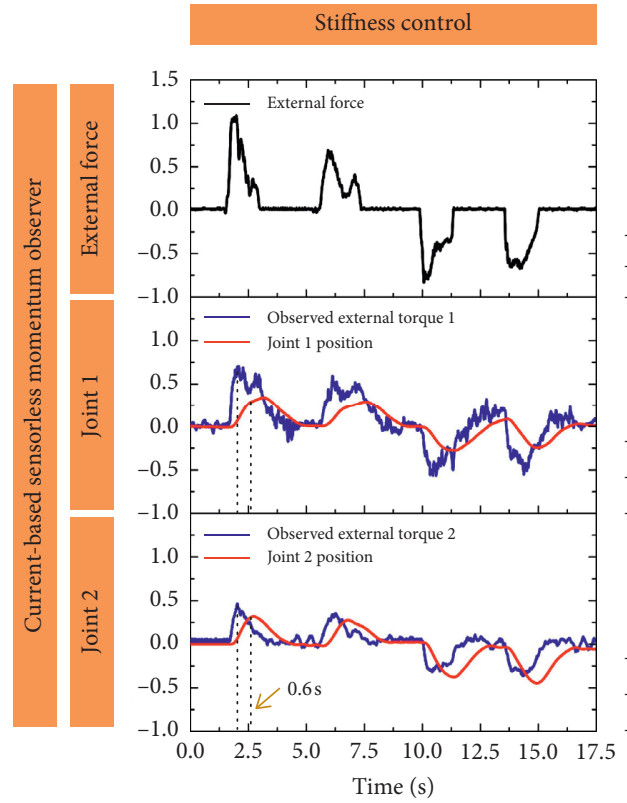

(d)

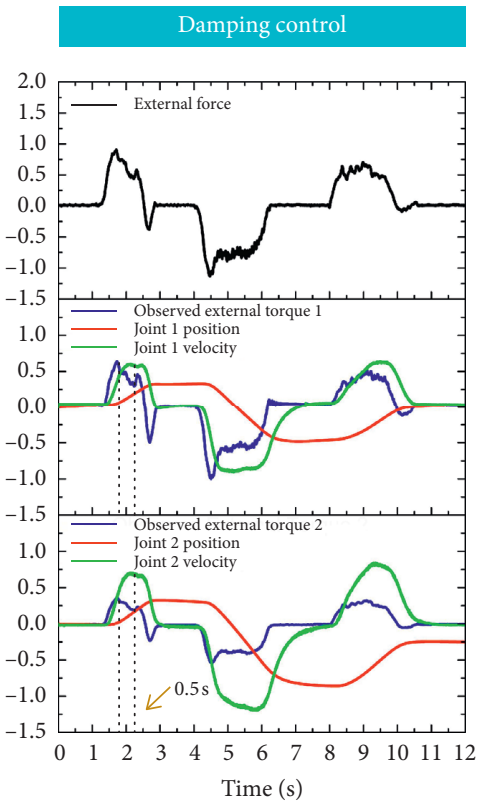

(b)

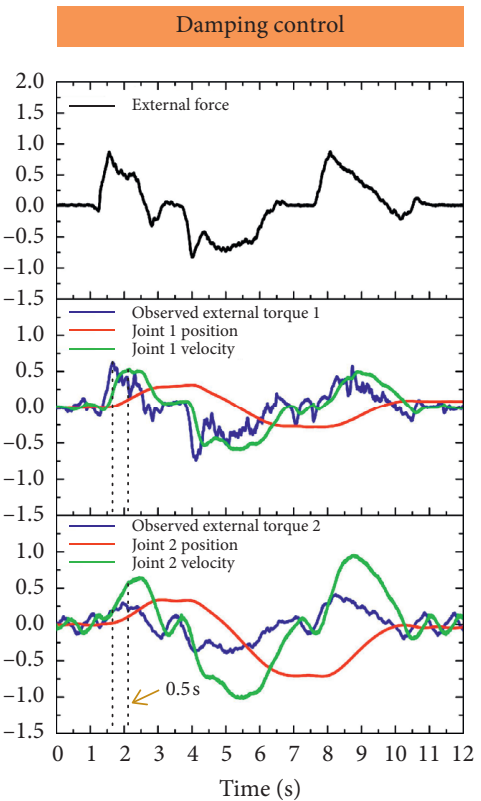

(e)

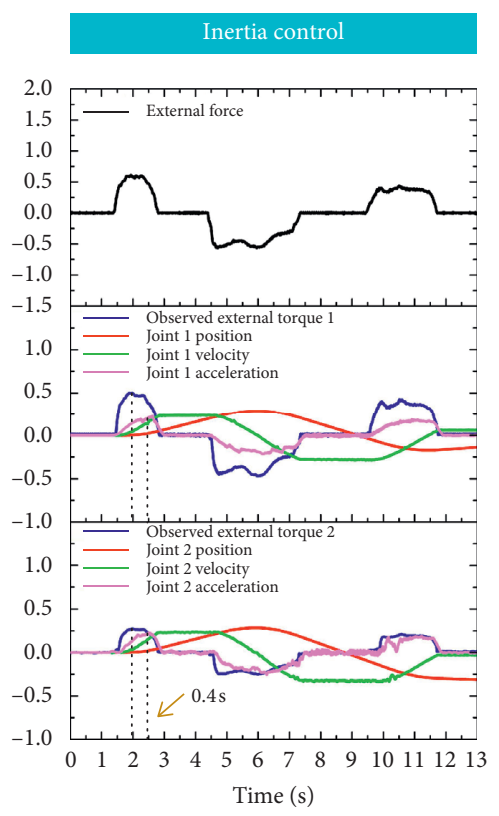

(c)

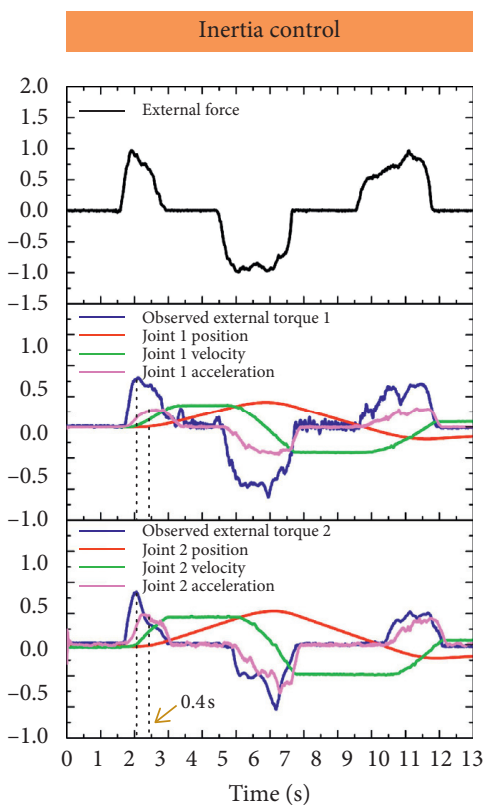

(f)

Figure 10: Normalized experiment results of stiffness, damping, and inertia controls based on momentum observer using torque sensors and current command on the 2-DOF manipulator. The normalization parameters are force: $20 \mathrm{~N}$; torque: $12 \mathrm{Nm}$; position: 1 rad; velocity: $0.5 \mathrm{rad} / \mathrm{s}$; and acceleration: $0.6 \mathrm{rad} / \mathrm{s}^{2}$.

and adjusts the convergence speed of compliance controller. For stiffness control, the response time of the actual position to the external torque was $0.6 \mathrm{~s}$. For damping control, the response time of the actual velocity to the external torque was $0.5 \mathrm{~s}$. For inertia control, the response time of the actual acceleration to the external torque was $0.4 \mathrm{~s}$. The response time is within an appropriate range.

The shortcoming of applying torque servo control for sensorless compliance control discussed in Section 4.3 was also eliminated by applying the position servo loop. Although the current-based observer brought errors of stiction, the mapping strategy reflected the error to joint motion. Therefore, the robot joint was always in slight motion (see example in Figure 10(e)), which changed the stiction state to the sliding state. Sliding friction is much lower than stiction. Thus, the external information was easier to be transmitted to the motor side. The operator did not have to overcome a large resistance to actuate the manipulator.

Experiments for three control methods were produced under different scale factors. The scale factors were set as $K_{P}^{i}=0.1,0.3,0.5,1.0(i=1,2)$ for stiffness control; $K_{Q}^{i}=0.3$, $0.5,1.0,1.5(i=1,2)$ for damping control; and $K_{R}^{i}=0.1,0.3$, $0.5,1.0(i=1,2)$ for inertia control. Figure 11 presents the 


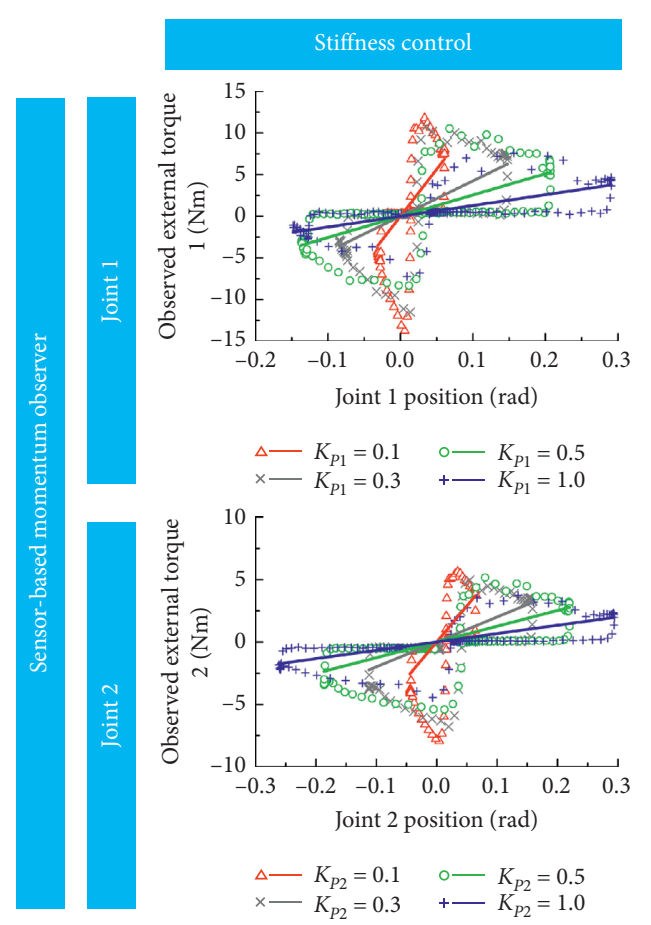

(a)

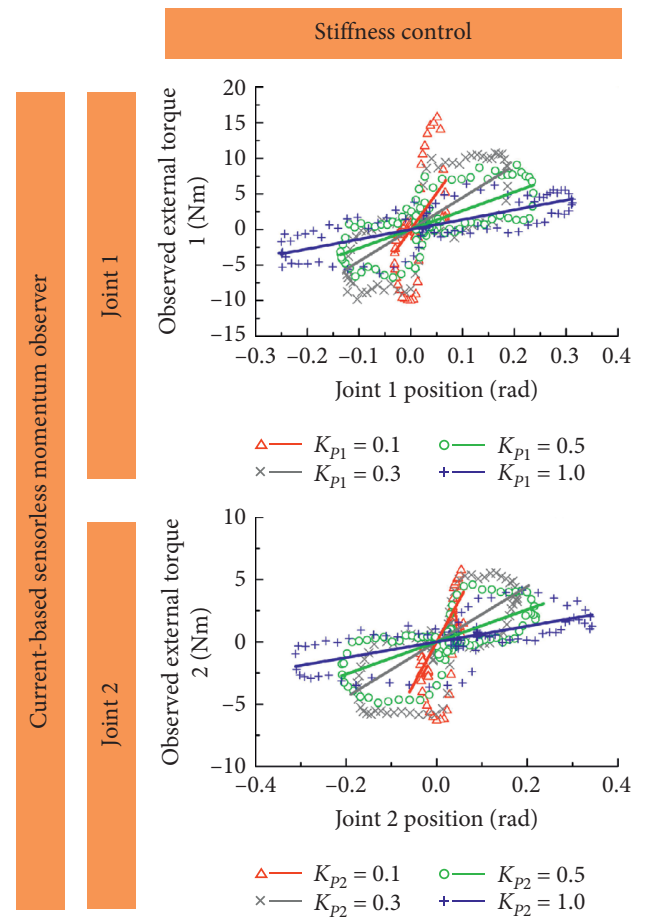

(d)
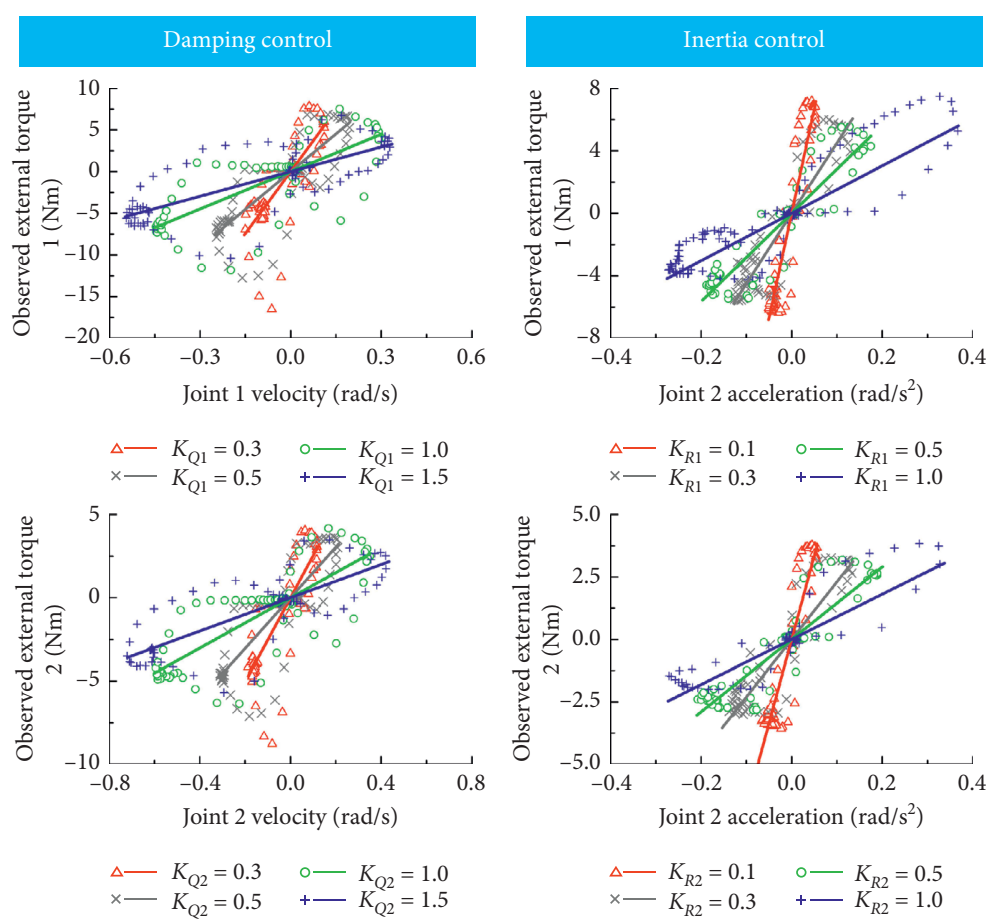

(b)
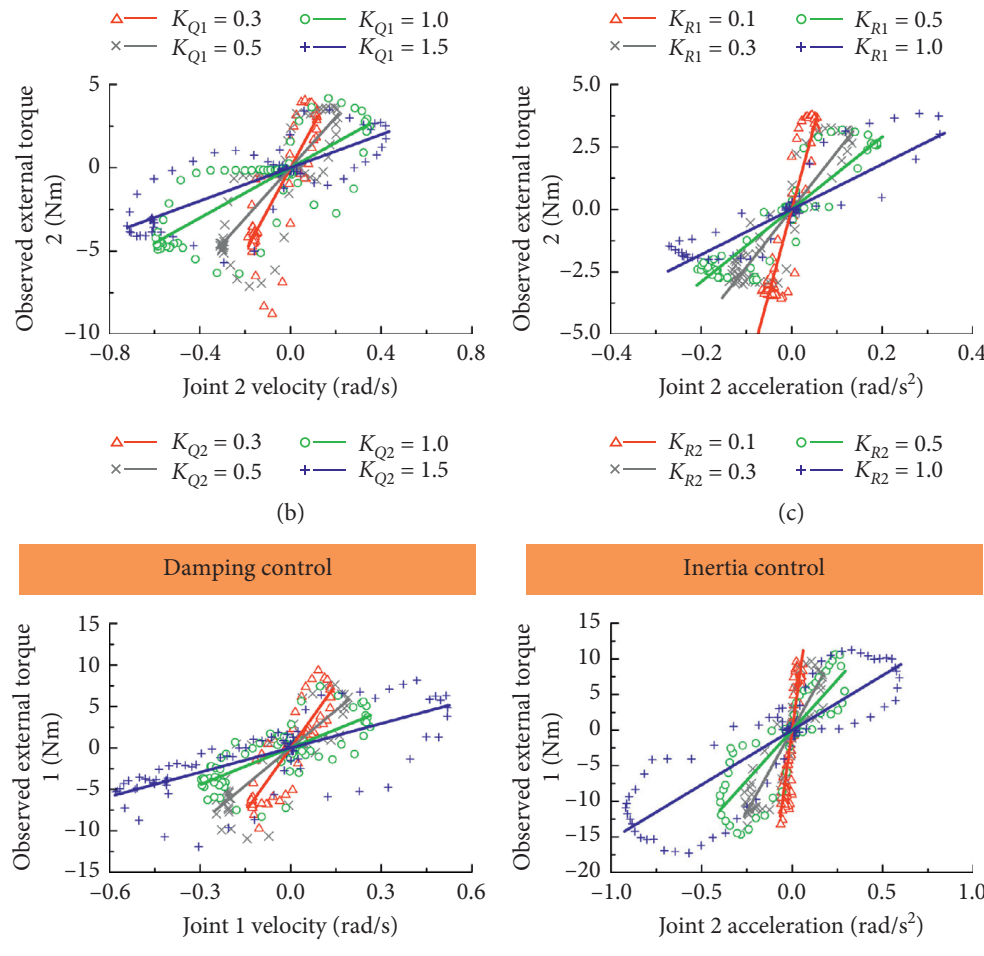

(c)
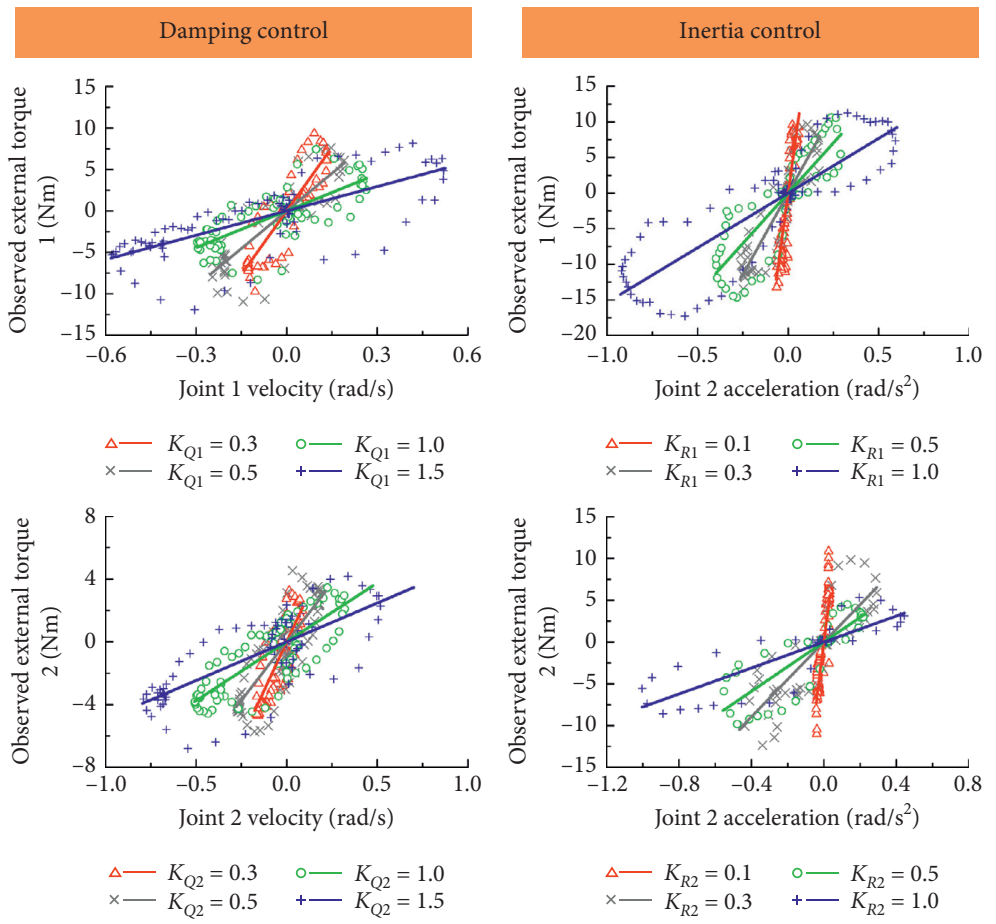

(e)

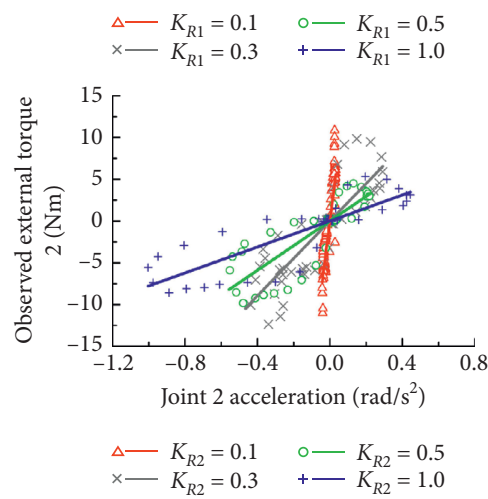

(f)

FIGURE 11: Virtual characteristic of three control methods based on sensor-based momentum observer and current-based observer under different characteristic scale factors.

virtual characteristics under different scale factor values. Linear fitting was produced to obtain the relationship between external torque and the recorded trajectory. The slope of fitted lines represented the virtual joint characteristics, including virtual stiffness, damping, and inertia, as shown in Figure 12. The previous discussion of equation
(27) was proven. The virtual compliance characteristics were proportional to $1 / K_{P}, 1 / K_{Q}$, and $1 / K_{R}$, and linear tuning was achieved by adjusting the scaling factors $K_{P}$, $K_{Q}$, and $K_{R}$. This feature of quantitative adjustability in joint space gave the manipulator a wide adjustable range on a single virtual feature. 


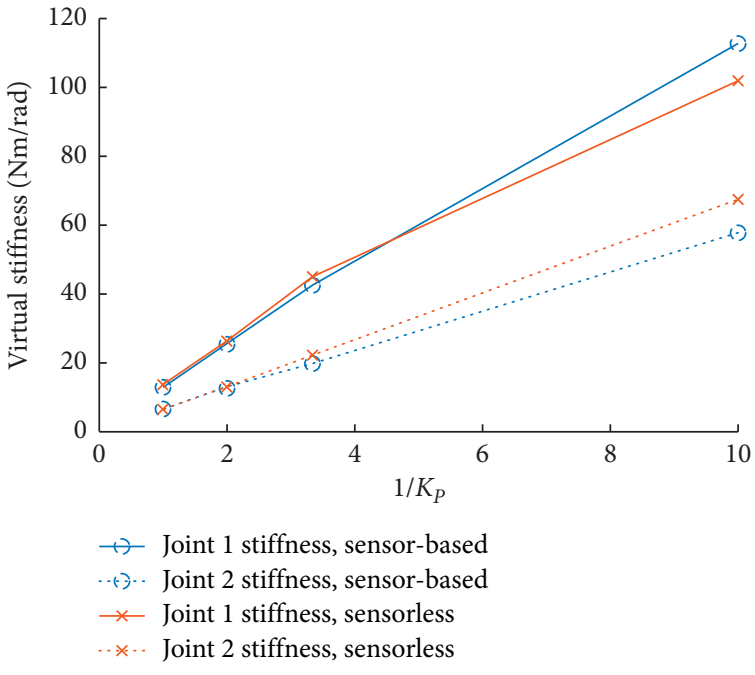

(a)

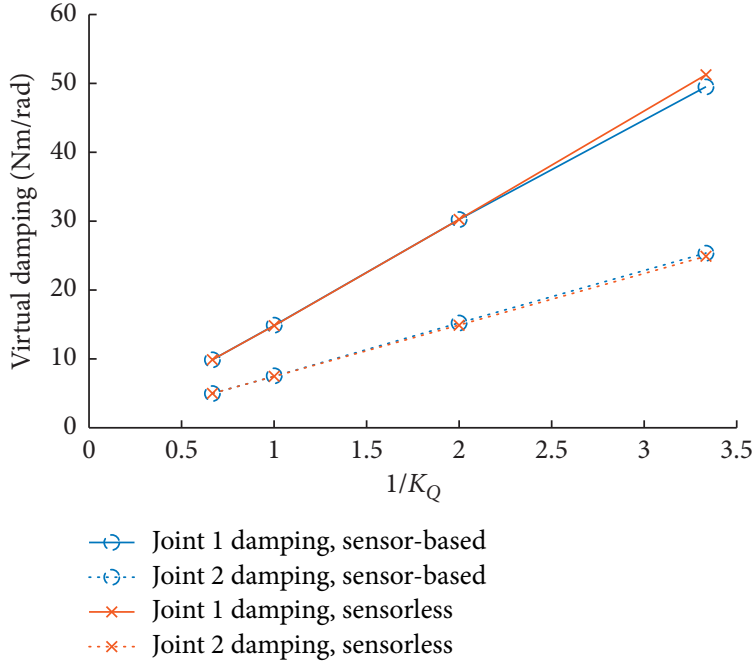

(b)

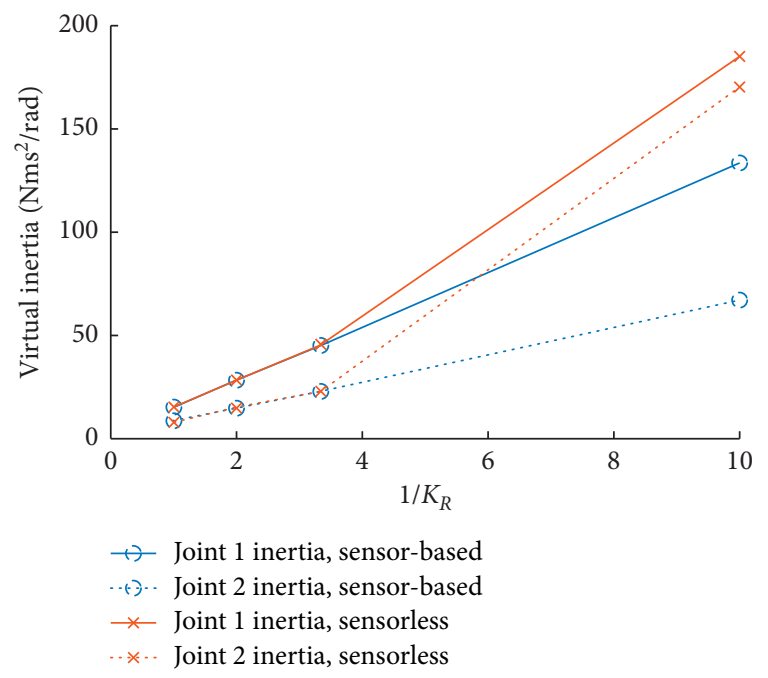

(c)

Figure 12: Virtual compliance feature under different scale factors. (a) Virtual stiffness. (b) Virtual damping. (c) Virtual inertia.

\section{Conclusion}

We propose a cost-effective whole-body compliance control scheme based on sensorless torque estimation and haptic filter. This strategy has a great potential in industrial low-cost collaborative manipulators. A modified sensorless momentum observer using motor current command is realized on the premise of considering manipulator-with-actuator dynamics. This observer is applied in the joint space compliance control. Virtual spring, damper, and inertia features can be achieved by stiffness, damping, and inertia control algorithm, which eliminates the necessity of using multiple control algorithms for collaborative robotic arms. The linear adjustment of each virtual haptic feature can be easily achieved by tuning a scale factor.

The effectiveness of the sensorless momentum observer and compliance control strategy is validated through comprehensive experiments. The performance using the current command is proven to be similar to the performance using the torque sensor. Other findings of experiments are as follows: (a) the stiction modeling affects the performance of the sensorless momentum observer when joints are under static status. (b) Direct mapping of current-based torque estimation on torque command causes unsmooth contact force during physical human-robot interaction, which is due to the uncertainty of stiction. Our experimental data support that the operator can still feel a natural haptic perception even if the friction compensation is not so rigorous by using the position controller in combination with the haptic filter.

The proposed algorithm can be applied on any kinds of rigid manipulator in theory as long as the robot dynamics are available. For the manipulators without torque sensors, only motor-side position feedback and back-drivability are required. For the manipulators with torque sensors, backdrivability can be ignored. The current control methodology has ignored model uncertainty that may lead to small inaccuracy on external torque estimation. The dynamic model of robot manipulator is a coupled nonlinear system. 
Thus, sliding mode control technique can be used to deal with the model uncertainty in the proposed controller $[32,42]$. The combination of proposed compliance control with integral sliding mode control to reject model uncertainties will be studied in the future. Furthermore, the friction model accuracy slightly affects the performance of sensorless control, especially when the joint velocity is low. We will focus on the study of friction identification and compensation for sensorless compliance control on the basis of the results of this research.

\section{Data Availability}

The raw/processed data required to reproduce these findings cannot be shared at this time as the data also form part of an ongoing study.

\section{Conflicts of Interest}

The authors declare that they have no conflicts of interest regarding the publication of this paper.

\section{Acknowledgments}

This work was supported by the National Key R\&D Program of China (Grant no. 2017YFF0108000).

\section{References}

[1] X. Liu, T. Zhang, J. Li, Y. Guan, and G. Liu, "A novel endeffector for robotic compliant polishing," in Proceedings of the 2018 IEEE International Conference on Robotics and Biomimetics (ROBIO), Kuala Lumpur, Malaysia, December 2018.

[2] L. Bascetta, G. Ferretti, G. Magnani, and P. Rocco, "Walkthrough programming for robotic manipulators based on admittance control," Robotica, vol. 31, no. 7, pp. 1143-1153, 2013.

[3] M. Ragaglia, A. M. Zanchettin, L. Bascetta, and P. Rocco, "Accurate sensorless lead-through programming for lightweight robots in structured environments," Robotics and Computer-Integrated Manufacturing, vol. 39, pp. 9-21, 2016.

[4] L. Han, W. Xu, B. Li, and P. Kang, "Collision detection and coordinated compliance control for a dual-arm robot without force/torque sensing based on momentum observer," IEEE/ ASME Transactions on Mechatronics, vol. 24, no. 5, pp. 2261-2272, 2019.

[5] H. Sadeghian, L. Villani, M. Keshmiri, and B. Siciliano, "Taskspace control of robot manipulators with null-space compliance," IEEE Transactions on Robotics, vol. 30, no. 2, pp. 493-506, 2014.

[6] X. Li, Y. Pan, G. Chen, and H. Yu, "Continuous tracking control for a compliant actuator with two-stage stiffness," IEEE Transactions on Automation Science and Engineering, vol. 15, no. 1, pp. 57-66, 2018.

[7] J. Luo, S. Wang, Y. Zhao, and Y. Fu, "Variable stiffness control of series elastic actuated biped locomotion," Intelligent Service Robotics, vol. 11, no. 3, pp. 225-235, 2018.

[8] E. Mariotti, E. Magrini, and A. De Luca, "Admittance control for human-robot interaction using an industrial robot equipped with a F/T sensor," in Proceedings of the 2019 International Conference on Robotics and Automation, pp. 6130-6136, Montreal, Canada, May 2019.
[9] Q. Leboutet, E. Dean-Leon, F. Bergner, and G. Cheng, "Tactile-based whole-body compliance with force propagation for mobile manipulators," IEEE Transactions on Robotics, vol. 35, no. 2, pp. 330-342, 2019.

[10] Q.-T. Chu, H. Tanaka, H. Inuzuka, Y. Morita, and M. Sakai, "Study on hybrid position/force teaching and control method for 6 DoF manipulator utilizing f-PAWTED," Journal of Robotics Networking and Artificial Life, vol. 7, pp. 222-226, 2021.

[11] A. Mifsud, M. Benallegue, and F. Lamiraux, "Stabilization of a compliant humanoid robot using only inertial measurement units with a viscoelastic reaction mass pendulum model," in Proceedings of the 2016 IEEE/RSJ International Conference on Intelligent Robots and Systems (IROS), Daejeon, Korea, October 2016.

[12] K. S. Eom, I. H. Suh, W. K. Chung, and S. Oh, "Disturbance observer based force control of robot manipulator without force sensor," in Proceedings of the 1998 IEEE International Conference on Robotics and Automation (Cat. No.98CH36146), pp. 3012-3017, Leuven, Belgium, May 1998.

[13] S. Morinaga and K. Kosuge, "Collision detection system for manipulator based on adaptive impedance control law," in Proceedings of the 2003 IEEE International Conference on Robotics and Automation (Cat. No. 03CH37422), pp. 1080-1085, Taipei, Taiwan, September 2003.

[14] A. D. Luca and R. Mattone, "Actuator failure detection and isolation using generalized momenta," in Proceedings of the 2003 IEEE International Conference on Robotics and Automation (Cat. No. 03CH37422), pp. 634-639, Taipei, Taiwan, September 2003.

[15] A. D. Luca, A. Albu-Schaffer, S. Haddadin, and G. Hirzinger, "Collision detection and safe reaction with the DLR-III lightweight manipulator arm," in Proceedings of the 2006 IEEE/RSJ International Conference on Intelligent Robots and Systems, pp. 1623-1630, Beijing, China, October 2006.

[16] A. Wahrburg, E. Morara, G. Cesari, B. Matthias, and H. Ding, "Cartesian contact force estimation for robotic manipulators using Kalman filters and the generalized momentum," in Proceedings of the 2015 International Conference on Automation Science and Engineering, pp. 1230-1235, Gothenburg, Sweden, August 2015.

[17] A. Wahrburg, J. Bös, K. D. Listmann, F. Dai, B. Matthias, and H. Ding, "Motor-current-based estimation of Cartesian contact forces and torques for robotic manipulators and its application to force control," IEEE Transactions on Automation Science and Engineering, vol. 15, no. 2, pp. 879-886, 2018.

[18] S. Lee, M. Kim, and J. Song, "Sensorless collision detection for safe human-robot collaboration," in Proceedings of the 2015 IEEE/RSJ International Conference on Intelligent Robots and Systems (IROS), pp. 2392-2397, Hamburg, Germany, September 2015.

[19] S. Haddadin, A. Albu-Schaffer, A. D. Luca, and G. Hirzinger, "Collision detection and reaction: a contribution to safe physical human-robot interaction," in Proceedings of the 2008 IEEE/RSJ International Conference on Intelligent Robots and Systems, pp. 3356-3363, Nice, France, September 2008.

[20] X. Liu, F. Zhao, S. S. Ge, Y. Wu, and X. Mei, "End-effector force estimation for flexible-joint robots with global friction approximation using neural networks," IEEE Transactions on Industrial Informatics, vol. 15, no. 3, pp. 1730-1741, 2019.

[21] M. J. Kim, Y. J. Park, and W. K. Chung, "Design of a momentum-based disturbance observer for rigid and flexible 
joint robots," Intelligent Service Robotics, vol. 8, no. 1, pp. 57-65, 2015.

[22] N. Hogan, "Impedance control: an approach to manipulation," in Proceedings of the 1984 American Control Conference, pp. 304-313, San Diego, CA, USA, 1984.

[23] H. Feng-Yih and F. Li-Chen, "Adaptive fuzzy hybrid force/ position control for robot manipulators following contours of an uncertain object," in Proceedings of the IEEE International Conference on Robotics and Automation, pp. 2232-2237, Minneapolis, MN, USA, April 1996.

[24] M. H. Raibert and J. J. Craig, "Hybrid position/force control of manipulators," Journal of Dynamic Systems, Measurement, and Control, vol. 103, no. 2, pp. 126-133, 1981.

[25] S. Chiaverini and L. Sciavicco, "The parallel approach to force/ position control of robotic manipulators," IEEE Transactions on Robotics and Automation, vol. 9, no. 4, pp. 361-373, 1993.

[26] P. Song, Y. Yu, and X. Zhang, "Impedance control of robots: an overview," in Proceedings of the 2017 2nd International Conference on Cybernetics, Robotics and Control (CRC), pp. 51-55, Chengdu, China, July 2017.

[27] M. Schumacher, J. Wojtusch, P. Beckerle, and O. von Stryk, "An introductory review of active compliant control," Robotics and Autonomous Systems, vol. 119, pp. 185-200, 2019.

[28] G. Peng, C. Yang, W. He, and C. L. P. Chen, "Force sensorless admittance control with neural learning for robots with actuator saturation," IEEE Transactions on Industrial Electronics, vol. 67, no. 4, pp. 3138-3148, 2020.

[29] B. Yao, Z. Zhou, L. Wang, W. Xu, Q. Liu, and A. Liu, "Sensorless and adaptive admittance control of industrial robot in physical human-robot interaction," Robotics and Computer-Integrated Manufacturing, vol. 51, pp. 158-168, 2018.

[30] D. Nicolis, F. Allevi, and P. Rocco, "Operational space model predictive sliding mode control for redundant manipulators," IEEE Transactions on Robotics, vol. 36, no. 4, pp. 1348-1355, 2020.

[31] X. Zhang, T. Sun, and D. Deng, "Neural approximation-based adaptive variable impedance control of robots," Transactions of the Institute of Measurement and Control, vol. 42, no. 13, pp. 2589-2598, 2020.

[32] Y. Wang, X. Xie, M. Chadli, S. Xie, and Y. Peng, "Sliding mode control of fuzzy singularly perturbed descriptor systems," IEEE Transactions on Fuzzy Systems, p. 1, 2020.

[33] F. Zeng, J. Xiao, and H. Liu, "Force/torque sensorless compliant control strategy for assembly tasks using a 6-DOF collaborative robot," IEEE Access, vol. 7, pp. 108795-108805, 2019.

[34] D. Tsetserukou, N. Kawakami, and S. Tachi, "Design, control and evaluation of a whole-sensitive robot arm for physical human-robot interaction," International Journal of Humanoid Robotics, vol. 6, no. 4, pp. 699-725, 2009.

[35] W. He, C. Xue, X. Yu, Z. Li, and C. Yang, "Admittance-based controller design for physical human-robot interaction in the constrained task space," IEEE Transactions on Automation Science and Engineering, vol. 17, no. 4, pp. 1937-1949, 2020.

[36] Y. Chen, J. Hu, W. Wang, L. Peng, L. Peng, and Z.-G. Hou, "An FES-assisted training strategy combined with impedance control for a lower limb rehabilitation robot," in Proceedings of the 2013 IEEE International Conference on Robotics and Biomimetics (ROBIO), pp. 2037-2042, Shenzhen, China, December 2013.

[37] M. Tufail and C. W. de Silva, "Impedance control schemes for bilateral teleoperation," in Proceedings of the 2014 9th
International Conference on Computer Science \& Education, pp. 44-49, Vancouver, Canada, August 2014.

[38] D. J. Latornell and D. B. Cherchas, "Force and motion control of a single flexible manipulator link," Robotics and ComputerIntegrated Manufacturing, vol. 9, no. 2, pp. 87-99, 1992.

[39] A. Flores-Abad, M. Nandayapa, and M. A. Garcia-Teran, "Force sensorless impedance control for a space robot to capture a satellite for on-orbit servicing," in Proceedings of the 2018 IEEE Aerospace Conference, pp. 1-7, Piscataway, NJ, USA, March 2018.

[40] S. Haddadin, A. De Luca, and A. Albu-Schäffer, "Robot collisions: a survey on detection, isolation, and identification," IEEE Transactions on Robotics, vol. 33, no. 6, pp. 1292-1312, 2017.

[41] B. Bona and M. Indri, "Friction compensation in robotics: an overview," in Proceedings of the 44th IEEE Conference on Decision and Control, pp. 4360-4367, Seville, Spain, December 2005.

[42] Y. Wang, Y. Xia, H. Li, and P. Zhou, "A new integral sliding mode design method for nonlinear stochastic systems," Automatica, vol. 90, pp. 304-309, 2018. 